\title{
THE INFLUENCE OF BOUNDARY DATA ON THE NUMBER OF SOLUTIONS OF BOUNDARY VALUE PROBLEMS WITH JUMPING NONLINEARITIES
}

\author{
GREG A. HARRIS
}

\begin{abstract}
This paper contains results concerning the number of solutions, as a function of the boundary data, for boundary value problems with jumping nonlinearities. An example seems to indicate that boundary data has a different influence on this number than does forcing data. Through approximating techniques this example leads to lower bounds on solution numbers for the more general case
\end{abstract}

\section{INTRODUCTION}

Consider the nonlinear boundary value problem

$$
\left\{\begin{array}{l}
u^{\prime \prime}(x)+f(u(x))=h(x), \\
u(0)=\sigma_{1}, \quad u(\pi)=\sigma_{2} .
\end{array}\right.
$$

Here $\sigma_{1}$ and $\sigma_{2}$ are prescribed real numbers and $f \in C(\mathbf{R} ; \mathbf{R})$ and has asymptotic limits

$$
\frac{f(s)}{s} \rightarrow \begin{cases}\alpha, & s \rightarrow-\infty, \\ \beta, & s \rightarrow \infty,\end{cases}
$$

for some real numbers $\alpha$ and $\beta$. Such $f$ have come to be called jumping nonlinearities, a term coined by Fučik [F1].

It is convenient to think of $f$ in the form

$$
f(s)=\alpha s^{-}+\beta s^{+}+\nu(s), \quad s \in \mathbf{R},
$$

where $\nu \in C(\mathbf{R} ; \mathbf{R})$ and

$$
|\nu(s) / s| \rightarrow 0 \text { as }|s| \rightarrow \infty .
$$

Here $(\cdot)^{+}: \mathbf{R} \rightarrow \mathbf{R}$ and $(\cdot)^{-}: \mathbf{R} \rightarrow \mathbf{R}$ are defined by $s^{+} \stackrel{\text { def }}{=} \max \{0, s\}$ and $s^{-} \stackrel{\text { def }}{=}$ $\min \{0, s\}$ respectively.

To get at lower bounds on the number of solutions of (1) consider $\nu$ a perturbation term and then look at the unperturbed problem

$$
\left\{\begin{array}{l}
u^{\prime \prime}(x)+\alpha u^{-}+\beta u^{+}=0, \\
u(0)=\sigma_{1}, \quad u(\pi)=\sigma_{2} .
\end{array}\right.
$$

Received by the editors September 28, 1988.

1980 Mathematics Subject Classification (1985 Revision). Primary 34B15; Secondary 34A35, $35 \mathrm{~B} 32$. 
Note the free parameters $\alpha, \beta, \sigma_{1}$ and $\sigma_{2}$.

Degree arguments can be used to extend results obtained for this problem to the perturbed problem (1).

Problems of this type have been popular in recent years. Further results in this direction may be found in Amann and Hess [AH], Dancer [Da1, Da2], Fučik [F1, F2, F3], Hess [He], Lazer and McKenna [LMc1, LMc2, LMc3], Schmitt [Sc1, Sc3], and Solimini [So].

In problem (2) we may assume, without loss of generality, that $\alpha \leq \beta$. For if $w$ solves (2) with $\beta<\alpha$ then, in view of the relationship $-\left(w^{+}\right)=(-w)^{-}$, $u \stackrel{\text { def }}{=}-w$ satisfies

$$
\left\{\begin{array}{l}
u^{\prime \prime}(x)+\beta u^{-}+\alpha u^{+}=0, \\
u(0)=-\sigma_{1}, \quad u(\pi)=-\sigma_{2} .
\end{array}\right.
$$

Precise results concerning the number of solutions of (2) will be obtained for each given pair of boundary conditions $\sigma_{1}$ and $\sigma_{2}$ and positive parameter values $\alpha$ and $\beta$. There is evidence that as the nonlinearity interacts with more of the spectrum, that is, as $\beta$ crosses over higher eigenvalues, then changing boundary data has a very different effect from changing forcing data on the number of solutions; i.e., if the pair $(\alpha, \beta)$ belong to certain well-defined regions of the parameter quadrant $Q^{+} \stackrel{\text { def }}{=}\{(\alpha, \beta) \mid \alpha>0, \beta>0\}$ then the unperturbed problem (2) has precisely zero, one, or two solutions depending on the choice of $\sigma_{1}$ and $\sigma_{2}$. It should be noted that these regions are unbounded in the $\beta$ direction and, as such, the result should be in contrast to what one might expect. That is, regardless of the number of eigenvalues $\beta$ crosses, the number of solutions of (2) never exceeds two.

\section{SimPLifYING THE SITUATION}

Problem (2) may be reduced to three cases: $\sigma_{1}=1, \sigma_{1}=0$, and $\sigma_{1}=-1$; i.e., if $\alpha$ and $\beta$ are fixed and if $u_{1}$ is a solution of (2) with $u_{1}(0)=\sigma_{1}>0$ and $u_{1}(\pi)=\sigma_{2}$, then $u_{2} \stackrel{\text { def }}{=} u_{1} / \sigma_{1}, \sigma_{1}>0$, is a solution of $(2)$ with $u_{2}(0)=1$ and $u_{2}(\pi)=\sigma_{2} / \sigma_{1}$. Conversely, if $u_{2}$ is a solution of (2) with $u_{2}(0)=1$ and $u_{2}(\pi)=\sigma_{2} / \sigma_{1}, \sigma_{1}>0$, then $u_{1} \stackrel{\text { def }}{=} \sigma_{1} u_{2}$ is a solution of $(2)$ with $u_{1}(0)=\sigma_{1}>0$ and $u_{1}(\pi)=\sigma_{2}$. A similar situation holds if $\sigma_{1}<0$.

Solutions of (2) may then be recovered from solutions of one of the following three problems:

$$
\begin{aligned}
& \left\{\begin{array}{l}
u^{\prime \prime}+\alpha u^{-}+\beta u^{+}=0, \\
u(0)=1, \quad u(\pi)=\sigma ;
\end{array}\right. \\
& \left\{\begin{array}{l}
u^{\prime \prime}+\alpha u^{-}+\beta u^{+}=0, \\
u(0)=0, \quad u(\pi)=\sigma ;
\end{array}\right. \\
& \begin{cases}u^{\prime \prime}+\alpha u^{-}+\beta u^{+}=0, \\
u(0)=-1, & u(\pi)=\sigma .\end{cases}
\end{aligned}
$$

These problems are analyzed below by determining, for fixed $\alpha$ and $\beta$, the 
range of the values of $\sigma$ for which each is solvable. This will be done by writing the differential equation

$$
u^{\prime \prime}+\alpha u^{-}+\beta u^{+}=0
$$

as the system

$$
\left\{\begin{array}{l}
\dot{u}=v, \\
\dot{v}=-\alpha u^{-}-\beta u^{+}
\end{array}\right.
$$

and then looking at the phase-plane structure of (7).

Indeed, if

$$
\left(\begin{array}{l}
u \\
v
\end{array}\right)\left[t ; \alpha, \beta,\left(\begin{array}{l}
u_{0} \\
v_{0}
\end{array}\right)\right]
$$

denotes the solution of (7) with initial condition

$$
\left(\begin{array}{l}
u_{0} \\
v_{0}
\end{array}\right)
$$

and $s$ is a positive real number, then setting

$$
\left(\begin{array}{l}
\bar{u} \\
\bar{v}
\end{array}\right)(t) \stackrel{\text { def }}{=} s\left(\begin{array}{l}
u \\
v
\end{array}\right)\left[t ; \alpha, \beta,\left(\begin{array}{l}
u_{0} \\
v_{0}
\end{array}\right)\right],
$$

we see that $\left(\frac{\bar{u}}{v}\right)(t)$ is a solution of the system (7) with initial condition

$$
s\left(\begin{array}{l}
u_{0} \\
v_{0}
\end{array}\right) .
$$

Trajectories of (7) will correspond to periodic orbits (if $\alpha>0$ ) about the origin, and hence if one nontrivial trajectory of (7) is known then, through scaling, they all are. Accordingly, all the information contained in system (7) may be recovered from any one nontrivial trajectory. One will be chosen that is convenient for our purposes, but first observe that system (7) is linear when restricted to either the left or the right half of the phase plane. That is, if $u, \leq 0$ the system takes the form

and if $u \geq 0$ the form

$$
\left\{\begin{array}{l}
\dot{u}=v \\
\dot{v}=-\alpha u
\end{array}\right.
$$

$$
\left\{\begin{array}{l}
\dot{u}=v \\
\dot{v}=-\beta u .
\end{array}\right.
$$

If $u_{0} \geq 0$ then the solution of (7) with initial condition

$$
\left(\begin{array}{l}
u_{0} \\
v_{0}
\end{array}\right)
$$

is given by

$$
\left(\begin{array}{l}
u \\
v
\end{array}\right)\left[t ;\left(\begin{array}{l}
u_{0} \\
v_{0}
\end{array}\right)\right]=\left(\begin{array}{cc}
\cos \sqrt{\beta} t & \frac{1}{\sqrt{\beta}} \sin \sqrt{\beta} t \\
-\sqrt{\beta} \sin \sqrt{\beta} t & \cos \sqrt{\beta} t
\end{array}\right)\left(\begin{array}{l}
u_{0} \\
v_{0}
\end{array}\right)
$$




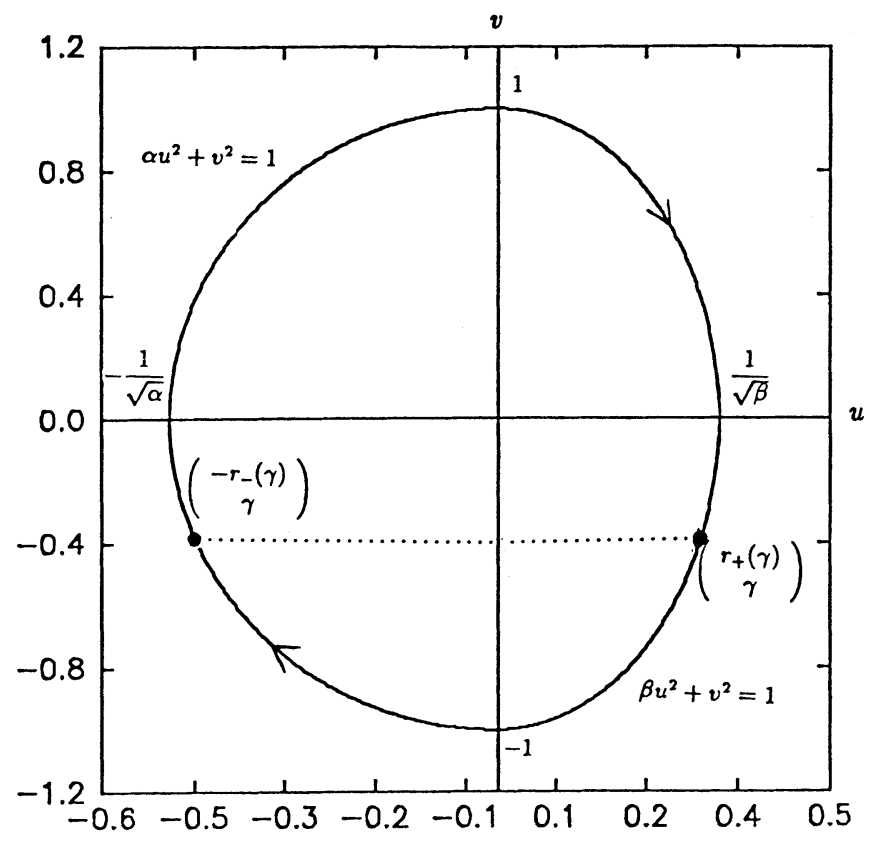

FIGURE 1. The periodic orbit of (7), with $(\alpha, \beta)=$ (4.9), through the point $\left(\begin{array}{l}0 \\ 1\end{array}\right)$

and is valid for as long as $u(t) \geq 0$. If

$$
\left(\begin{array}{l}
u_{0} \\
v_{0}
\end{array}\right) \in \mathbf{R}^{2}
$$

with $u_{0} \leq 0$ then

$$
\left(\begin{array}{l}
u \\
v
\end{array}\right)\left[t ;\left(\begin{array}{l}
u_{0} \\
v_{0}
\end{array}\right)\right]=\left(\begin{array}{cc}
\cos \sqrt{\alpha} t & \frac{1}{\sqrt{\alpha}} \sin \sqrt{\alpha} t \\
-\sqrt{\alpha} \sin \sqrt{\alpha} t & \cos \sqrt{\alpha} t
\end{array}\right)\left(\begin{array}{l}
u_{0} \\
v_{0}
\end{array}\right)
$$

is the solution of (7) with initial condition

$$
\left(\begin{array}{l}
u_{0} \\
v_{0}
\end{array}\right)
$$

and, again, is valid for as long as $u(t) \leq 0$.

The convenient trajectory for us will be the one passing through the point $\left(\begin{array}{l}0 \\ 1\end{array}\right)$. From (8) and (9) we obtain this trajectory by pasting together the following two solutions:

$$
\left(\begin{array}{l}
u \\
v
\end{array}\right)\left[t ;\left(\begin{array}{l}
0 \\
1
\end{array}\right)\right]=\left(\begin{array}{c}
\frac{1}{\sqrt{\beta}} \sin \sqrt{\beta} t \\
\cos \sqrt{\beta} t
\end{array}\right), \quad 0 \leq t \leq \frac{\pi}{\sqrt{\beta}},
$$

and

$$
\left(\begin{array}{l}
u \\
v
\end{array}\right)\left[t ;\left(\begin{array}{c}
0 \\
-1
\end{array}\right)\right]=\left(\begin{array}{c}
-\frac{1}{\sqrt{\alpha}} \sin \sqrt{\alpha} t \\
-\cos \sqrt{\alpha} t
\end{array}\right), \quad 0 \leq t \leq \frac{\pi}{\sqrt{\alpha}},
$$

which results in the periodic orbit shown in Figure 1. 
We denote this solution by $u\left[t ; \alpha, \beta,\left(\begin{array}{l}0 \\ 1\end{array}\right)\right]$ and see that it has minimal pe$\operatorname{riod} \pi / \sqrt{\alpha}+\pi / \sqrt{\beta}$. Evidently, then, all solutions of (7) may be obtained through a suitable time shift and scale of $\left(\begin{array}{l}u \\ v\end{array}\right)\left[t ; \alpha, \beta,\left(\begin{array}{l}0 \\ 1\end{array}\right)\right]$ and for notational convenience we denote this solution by

$$
\left(\begin{array}{l}
u \\
v
\end{array}\right)\left[t ;\left(\begin{array}{l}
u_{0} \\
v_{0}
\end{array}\right)\right]
$$

with the understanding that this solution depends continuously on the parameters $\alpha$ and $\beta$.

\section{A SHOOTING PROCEDURE}

We approach the problems (3), (4), and (5) by a simple shooting technique. A solution

$$
\left(\begin{array}{c}
\bar{u} \\
\bar{v}
\end{array}\right)\left[t ;\left(\begin{array}{l}
u_{0} \\
v_{0}
\end{array}\right)\right]
$$

will be started at time $t=0$, where $u_{0}$ is either 1,0 , or -1 , depending on which problem (3), (4), or (5), respectively, is under consideration; it is followed for a time interval of duration $\pi$ and there the value of $\bar{u}$, i.e.,

$$
\bar{u}\left[\pi ;\left(\begin{array}{c}
u_{0} \\
v_{0}
\end{array}\right)\right],
$$

is noted. The initial slope, $v_{0}$, is the shooting parameter and

$$
\sigma=\bar{u}\left[\pi ;\left(\begin{array}{l}
u_{0} \\
v_{0}
\end{array}\right)\right]
$$

is a $\sigma$ value for which the problem has a solution.

Starting a solution at an arbitrary point on the periodic orbit in Figure 1 will, in general, not satisfy the condition that $u_{0}$ be either 1,0 , or -1 . This matter is taken care of by the following scaling procedure.

Fix $\gamma \in(-1,1)$ and define

$$
r_{+}(\gamma) \stackrel{\text { def }}{=}(1 / \sqrt{\beta}) \sqrt{1-\gamma^{2}} \text { and } r_{-}(\gamma) \stackrel{\text { def }}{=}(1 / \sqrt{\alpha}) \sqrt{1-\gamma^{2}} .
$$

The two points

$$
\left(\begin{array}{c}
r_{+}(\gamma) \\
\gamma
\end{array}\right) \text { and }\left(\begin{array}{c}
-r_{-}(\gamma) \\
\gamma
\end{array}\right)
$$

lie on the orbit as indicated in Figure 1 and the solution

$$
\left(\begin{array}{l}
u \\
v
\end{array}\right)\left[t ;\left(\begin{array}{c}
r_{+}(\gamma) \\
\gamma
\end{array}\right)\right]
$$

then satisfies the differential equation (6) with

$$
u\left[0 ;\left(\begin{array}{c}
r_{+}(\gamma) \\
\gamma
\end{array}\right)\right]=r_{+}(\gamma)>0 .
$$

We want this solution to satisfy $u(0)=1$ and so define

$$
\left(\begin{array}{c}
u_{+} \\
v_{+}
\end{array}\right)[t ; \gamma] \stackrel{\text { def }}{=} \frac{1}{r_{+}(\gamma)}\left(\begin{array}{c}
u \\
v
\end{array}\right)\left[t ;\left(\begin{array}{c}
r_{+}(\gamma) \\
\gamma
\end{array}\right)\right], \quad t \in \mathbf{R} .
$$


$u_{+}[t ; \gamma]$ is then a solution of $(6)$ with $u_{+}[0 ; \gamma]=1$ and thus a solution of $(3)$ with $\sigma=u_{+}[\pi ; \gamma]$.

Setting

$$
\sigma_{+}(\gamma) \stackrel{\text { def }}{=} u_{+}[\pi ; \gamma], \quad-1<\gamma<1,
$$

observe that the range of $\sigma$-values for which (3) has a solution is just the range of the function $\sigma_{+}:(-1,1) \rightarrow \mathbf{R}$.

Problem (5) is treated in the same fashion. For $-1<\gamma<1$ define

$$
\left(\begin{array}{l}
u_{-} \\
v_{-}
\end{array}\right)[t ; \gamma] \stackrel{\text { def }}{=}\left(\begin{array}{l}
u \\
v
\end{array}\right)\left[t ;\left(\begin{array}{c}
-r_{-}(\gamma) \\
\gamma
\end{array}\right)\right], \quad t \in \mathbf{R},
$$

and let

$$
\sigma_{-}(\gamma) \stackrel{\text { def }}{=} u_{-}[\pi ; \gamma] \text {. }
$$

Then the range of $\sigma$ values for which (5) is solvable may be determined by the range of the function $\sigma_{-}:(-1,1) \rightarrow \mathbf{R}$.

Problem (4) is handled by choosing $\gamma$ to be either 1 or -1 (i.e., so that $u(0)=0)$. More is said concerning this situation later, but one can already see that if

$$
u\left[\pi ;\left(\begin{array}{l}
0 \\
1
\end{array}\right)\right] u\left[\pi ;\left(\begin{array}{c}
0 \\
-1
\end{array}\right)\right]<0
$$

then (4) has a solution for each $\sigma \in \mathbf{R}$. If

$$
u\left[\pi ;\left(\begin{array}{l}
0 \\
1
\end{array}\right)\right] u\left[\pi ;\left(\begin{array}{c}
0 \\
-1
\end{array}\right)\right]>0
$$

then (4) is solvable for all $\sigma \geq 0$ or all $\sigma \leq 0$ depending on whether the signs of the individual terms in the above product are negative or positive, respectively.

If it is the case that

$$
u\left[\pi ;\left(\begin{array}{l}
0 \\
1
\end{array}\right)\right] u\left[\pi ;\left(\begin{array}{c}
0 \\
-1
\end{array}\right)\right]=0
$$

then at least one of the values $u\left[\pi ;\left(\begin{array}{l}0 \\ 1\end{array}\right)\right]$ and $u\left[\pi ;\left(\begin{array}{c}0 \\ -1\end{array}\right)\right]$ must vanish and we have a nontrivial solution of

$$
\left\{\begin{array}{l}
u^{\prime \prime}+\alpha u^{-}+\beta u^{+}=0 \\
u(0)=u(\pi)=0
\end{array}\right.
$$

Of course, the conditions (17), (18), and (19), as well as the graphs of the functions $\sigma_{-}$and $\sigma_{+}$, will depend on how the positive parameters $\alpha$ and $\beta$ are chosen. As will be seen later, each of conditions (17), (18), and (19) is satisfied for certain values of $\alpha$ and $\beta$. In fact, the values of $\alpha$ and $\beta$ for which condition (19) is satisfied will be found to lie on curves separating the quadrant,

$$
Q^{+} \stackrel{\text { def }}{=}\{(\alpha, \beta) \mid \alpha>0, \beta>0\}
$$


into regions where either condition (17) or condition (18) holds and in which, at least qualitatively, the graphs of $\sigma_{-}$are the same, as well as the graphs of the functions $\sigma_{+}$. These graphs are used to get at the number of solutions of problem (3) and problem (5).

\section{Parameter Regions}

It is known (cf. [F1]) that the homogeneous problem (20) has nontrivial solutions if and only if the pair $(\alpha, \beta) \in Q^{+}$is on one of the curves

$$
\begin{gathered}
(j+1) / \sqrt{\alpha}+(j+1) / \sqrt{\beta}=1, \\
j / \sqrt{\alpha}+(j+1) / \sqrt{\beta}=1, \\
(j+1) / \sqrt{\alpha}+j / \sqrt{\beta}=1
\end{gathered}
$$

for $j=0,1,2, \ldots$ We should remark that this situation is easily seen using the trajectory in Figure 1. That is, the solution $\left(\begin{array}{l}u \\ v\end{array}\right)\left[t ;\left(\begin{array}{l}0 \\ 1\end{array}\right)\right]$ of $(7)$ in Figure 1 has the property that $u\left[0 ;\left(\begin{array}{l}0 \\ 1\end{array}\right)\right]=0$. In view of the fact that the time it takes for such a solution to traverse first through the right half and then through the left half of the $u v$-plane is $\pi / \sqrt{\beta}$ and $\pi / \sqrt{\alpha}$ respectively, $u\left[\pi ;\left(\begin{array}{l}0 \\ 1\end{array}\right)\right]$ will vanish if and only if either

$$
\pi=(j+1) \pi / \sqrt{\alpha}+(j+1) \pi / \sqrt{\beta}
$$

or

$$
\pi=j \pi / \sqrt{\alpha}+(j+1) \pi / \sqrt{\beta}
$$

for some $j=0,1,2, \ldots$, i.e., if and only if $(\alpha, \beta)$ is on a curve in $Q^{+}$ defined by either (22) or (23). The other possibility is to start a solution of (7) at the point $\left(\begin{array}{c}0 \\ -1\end{array}\right)$. This will lead to a solution of $(20)$ if and only if $(\alpha, \beta)$ lies on one of the curves in $Q^{+}$described by either (22) or (24). We have sketched a few of these curves in Figure 2.

Fix $k \in\{0,1,2,3, \ldots\}$ and define the regions

$$
R(I) \stackrel{\text { def }}{=}\left\{(\alpha, \beta) \in Q^{+} \mid \alpha<\beta ; \frac{k}{\sqrt{\alpha}}+\frac{k}{\sqrt{\beta}}<1 ; \frac{k}{\sqrt{\alpha}}+\frac{k+1}{\sqrt{\beta}}>1\right\}
$$

if $k \geq 1$ or, in the case that $k=0$,

$$
\begin{aligned}
& R(I) \stackrel{\text { def }}{=}\left\{(\alpha, \beta) \in Q^{+} \mid 0<\alpha<\beta<1\right\}, \\
& R(I I) \stackrel{\text { def }}{=}\left\{(\alpha, \beta) \in Q^{+} \mid \frac{k}{\sqrt{\alpha}}+\frac{k+1}{\sqrt{\beta}}<1 ; \frac{k+1}{\sqrt{\alpha}}+\frac{k}{\sqrt{\beta}}>1\right\}, \\
& R(I I I) \stackrel{\text { def }}{=}\left\{(\alpha, \beta) \in Q^{+} \mid \alpha<\beta ; \frac{k+1}{\sqrt{\alpha}}+\frac{k}{\sqrt{\beta}}<1 ; \frac{k+1}{\sqrt{\alpha}}+\frac{k+1}{\sqrt{\beta}}>1\right\} .
\end{aligned}
$$

These regions are indicated in Figure 2 in the case $k=1$. If $(\alpha, \beta)$ belongs to any of them then, of course, the completely homogenous problem (20) has only the trivial solution. 


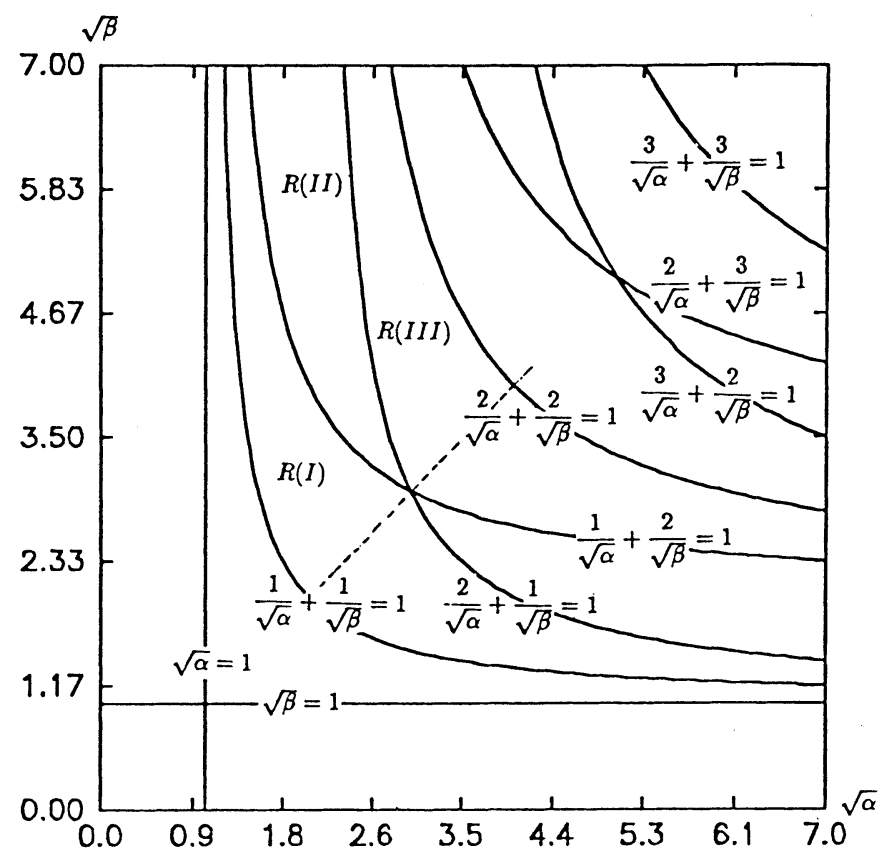

FIGURE 2. Parameter curves of the homogeneous problem (20)

\section{The RANGE OF $\sigma_{+}$AND $\sigma_{-}$}

Now the solution structure of problems (3) and (5) will be examined by, at least qualitatively, graphing the associated functions

$$
\sigma_{+}:(-1,1) \rightarrow \mathbf{R} \text { and } \sigma_{-}:(-1,1) \rightarrow \mathbf{R},
$$

where, from (13) and (14),

$$
\sigma_{+}(\gamma)=\frac{1}{r_{+}(\gamma)} u\left[\pi ;\left(\begin{array}{c}
r_{+}(\gamma) \\
\gamma
\end{array}\right)\right]
$$

and, from (15) and (16),

$$
\sigma_{-}(\gamma)=\frac{1}{r_{-}(\gamma)} u\left[\pi ;\left(\begin{array}{c}
-r_{-}(\gamma) \\
\gamma
\end{array}\right)\right] .
$$

It is convenient to remove, by way of a time shift, the shooting parameter $\gamma$ (i.e., the initial slope) from initial conditions in (26) and (27). To find the correct shift we compute the time $T_{+}$it takes for a solution of system (7) to move from the point $\left(\begin{array}{c}1 / \sqrt{\beta} \\ 0\end{array}\right)$ to the point $\left({ }^{r_{+}(\gamma)}\right)$ and then, correspondingly, the time $T_{-}$it takes a solution to move from $\left(\begin{array}{c}-1 / \sqrt{\alpha} \\ 0\end{array}\right)$ to $\left({ }^{-r_{-}(\gamma)}\right)$. In the case of $T_{+}$the part of the trajectory of concern belongs to the right half of the 
$u v$-plane (see Figure 1). Here $\dot{v}=-\beta u$ and hence the value of $T_{+}$is given by

$$
T_{+}=\int_{0}^{\gamma} \frac{d v}{-\beta u}=-\frac{1}{\sqrt{\beta}} \int_{0}^{\gamma} \frac{d v}{\sqrt{1-v^{2}}}=-\frac{1}{\sqrt{\beta}} \sin ^{-1} \gamma .
$$

Now consider the solution, $\left(\begin{array}{c}u \\ v\end{array}\right)\left[t ;\left(\begin{array}{c}0 \\ -1\end{array}\right)\right]$, of system (7). This solution has the initial condition $\left(\begin{array}{c}0 \\ -1\end{array}\right)$ and after time $t=-(\pi / 2 \sqrt{\beta})+T_{+}$we find that

$$
\left(\begin{array}{l}
u \\
v
\end{array}\right)\left[-\frac{\pi}{2 \sqrt{\beta}}-\frac{1}{\sqrt{\beta}} \sin ^{-1} \gamma ;\left(\begin{array}{c}
0 \\
-1
\end{array}\right)\right]=\left(\begin{array}{c}
r_{+}(\gamma) \\
\gamma
\end{array}\right) \text {. }
$$

That is, $-(\pi / 2 \sqrt{\beta})+T_{+}$is the time required for this solution to first back up to the point $\left(\begin{array}{c}1 / \sqrt{\beta} \\ 0\end{array}\right)$ and then move to the point $\left(c_{+}^{r_{\gamma}(\gamma)}\right)$ (again see Figure 1). Evidently, then for fixed $\alpha$ and $\beta$ and for each $-1 \leq \gamma \leq 1$ we have (28)

$$
\left(\begin{array}{l}
u \\
v
\end{array}\right)\left[t-\frac{\pi}{2 \sqrt{\beta}}-\frac{1}{\sqrt{\beta}} \sin ^{-1} \gamma ;\left(\begin{array}{c}
0 \\
-1
\end{array}\right)\right]=\left(\begin{array}{l}
u \\
v
\end{array}\right)\left[t ;\left(\begin{array}{c}
r_{+}(\gamma) \\
\gamma
\end{array}\right)\right], \quad t \in \mathbf{R} .
$$

The argument for $T_{-}$is similar. We find that

$$
T_{-}=(1 / \sqrt{\alpha}) \sin ^{-1} \gamma, \quad-1 \leq \gamma \leq 1,
$$

and so

$$
\left(\begin{array}{l}
u \\
v
\end{array}\right)\left[t-\frac{\pi}{2 \sqrt{\alpha}}+\frac{1}{\sqrt{\alpha}} \sin ^{-1} \gamma ;\left(\begin{array}{l}
0 \\
1
\end{array}\right)\right]=\left(\begin{array}{l}
u \\
v
\end{array}\right)\left[t ;\left(\begin{array}{c}
-r_{-}(\gamma) \\
\gamma .
\end{array}\right)\right], \quad t \in \mathbf{R} .
$$

For computational purposes make the change of variable

$$
\gamma=\sin \theta, \quad-\pi / 2 \leq \theta \leq \pi / 2 .
$$

The point $\left({ }^{r_{+}(\gamma)}{ }_{\gamma}\right)$ then becomes $\left(\frac{(1 / \sqrt{\beta}) \cos \theta}{\sin \theta}\right), T_{+}=T_{+}(\theta)$ may be written as

$$
T_{+}(\theta)=-(1 / \sqrt{\beta}) \theta, \quad-\pi / 2 \leq \theta \leq \pi / 2,
$$

and (28) becomes

$$
\left(\begin{array}{l}
u \\
v
\end{array}\right)\left[t-\frac{\pi}{2 \sqrt{\beta}}-\frac{1}{\sqrt{\beta}} \theta ;\left(\begin{array}{c}
0 \\
-1
\end{array}\right)\right]=\left(\begin{array}{l}
u \\
v
\end{array}\right)\left[t ;\left(\begin{array}{c}
(1 / \sqrt{\beta}) \cos \theta \\
\sin \theta
\end{array}\right)\right],
$$

for $t \in \mathbf{R}$ and $-\pi / 2 \leq \theta \leq \pi / 2$.

Similarly $\left({ }^{-r_{-}(\gamma)}\right)$ becomes $(\stackrel{-(1 / \sqrt{\alpha}) \cos \theta}{\sin \theta}), T_{-}(\theta)=(1 / \sqrt{\alpha}) \theta$, and (29) may be written as

$$
\begin{aligned}
\left(\begin{array}{l}
u \\
v
\end{array}\right)\left[t-\frac{\pi}{2 \sqrt{\alpha}}+\frac{1}{\sqrt{\alpha}} \theta ;\left(\begin{array}{l}
0 \\
1
\end{array}\right)\right] & \\
= & \left(\begin{array}{l}
u \\
v
\end{array}\right)\left[t ;\left(\begin{array}{c}
-(1 / \sqrt{\alpha}) \cos \theta \\
\sin \theta
\end{array}\right)\right], \quad t \in \mathbf{R}, \theta \in\left[-\frac{\pi}{2}, \frac{\pi}{2}\right] .
\end{aligned}
$$


Finally, since each of the solutions of system (7) has period $\pi / \sqrt{\alpha}+\pi / \sqrt{\beta}$, the following two expressions hold for each $j=0,1,2, \ldots, t \in \mathbf{R}$, and $-\pi / 2 \leq \theta \leq \pi / 2$.

$$
\left(\begin{array}{l}
u \\
v
\end{array}\right)\left[t-\pi\left(\frac{j}{\sqrt{\alpha}}+\frac{j}{\sqrt{\beta}}\right)-\frac{\pi}{2 \sqrt{\beta}}-\frac{1}{\sqrt{\beta}} \theta ;\left(\begin{array}{c}
0 \\
-1
\end{array}\right)\right]=\left(\begin{array}{l}
u \\
v
\end{array}\right)\left[t ;\left(\begin{array}{c}
\frac{1}{\sqrt{\beta}} \cos \theta \\
\sin \theta
\end{array}\right)\right],
$$

$$
\left(\begin{array}{l}
u \\
v
\end{array}\right)\left[t-\pi\left(\frac{j}{\sqrt{\alpha}}+\frac{j}{\sqrt{\beta}}\right)-\frac{\pi}{2 \sqrt{\alpha}}+\frac{1}{\sqrt{\alpha}} \theta ;\left(\begin{array}{l}
0 \\
1
\end{array}\right)\right]=\left(\begin{array}{l}
u \\
v
\end{array}\right)\left[t ;\left(\begin{array}{c}
-\frac{1}{\sqrt{\alpha}} \cos \theta \\
\sin \theta
\end{array}\right)\right] \text {. }
$$

5.1. For a fixed nonnegative integer $k$ and for $\theta \in[-\pi / 2, \pi / 2]$ define the two maps (times)

$$
\begin{aligned}
& \tau_{+}(\alpha, \beta, \theta) \stackrel{\text { def }}{=} \pi-\pi\left(\frac{k}{\sqrt{\alpha}}+\frac{k}{\sqrt{\beta}}\right)-\frac{\pi}{2 \sqrt{\beta}}-\frac{1}{\sqrt{\beta}} \theta, \\
& \tau_{-}(\alpha, \beta, \theta) \stackrel{\text { def }}{=} \pi-\pi\left(\frac{k}{\sqrt{\alpha}}+\frac{k}{\sqrt{\beta}}\right)-\frac{\pi}{2 \sqrt{\alpha}}+\frac{1}{\sqrt{\alpha}} \theta .
\end{aligned}
$$

Setting $t=\pi$ in (30) and (31) gives

$$
\begin{aligned}
\left(\begin{array}{l}
u \\
v
\end{array}\right)\left[\tau_{+}(\alpha, \beta, \theta) ;\left(\begin{array}{c}
0 \\
-1
\end{array}\right)\right] & =\left(\begin{array}{l}
u \\
v
\end{array}\right)\left[\pi ;\left(\begin{array}{c}
(1 / \sqrt{\beta}) \cos \theta \\
\sin \theta
\end{array}\right)\right], \\
\left(\begin{array}{l}
u \\
v
\end{array}\right)\left[\tau_{-}(\alpha, \beta, \theta) ;\left(\begin{array}{l}
0 \\
1
\end{array}\right)\right] & =\left(\begin{array}{l}
u \\
v
\end{array}\right)\left[\pi ;\left(\begin{array}{c}
-(1 / \sqrt{\alpha}) \cos \theta \\
\sin \theta
\end{array}\right)\right],
\end{aligned}
$$

and hence, from (26) and (27),

$$
\begin{array}{ll}
\sigma_{+}(\alpha, \beta, \theta)=\frac{u\left[\tau_{+}(\alpha, \beta, \theta) ;\left(\begin{array}{c}
0 \\
-1
\end{array}\right)\right]}{(1 / \sqrt{\beta}) \cos \theta}, & -\frac{\pi}{2}<\theta<\frac{\pi}{2}, \\
\sigma_{-}(\alpha, \beta, \theta)=\frac{u\left[\tau_{-}(\alpha, \beta, \theta) ;\left(\begin{array}{c}
0 \\
1
\end{array}\right)\right]}{(1 / \sqrt{\alpha}) \cos \theta}, & -\frac{\pi}{2}<\theta<\frac{\pi}{2} .
\end{array}
$$

It is the range of each of these two functions of $\theta$ that we wish to look at. Their dependence on the parameters $\alpha$ and $\beta$ is stressed by including them as arguments.

The arguments contained in the next few sections are to support some important qualitative features of these ranges that can be seen in the sample of graphs of $\sigma_{ \pm}$found in Figures 3 to 14. In these figures the two functions $\sigma_{+}(\alpha, \beta, \cdot)$ and $\sigma_{-}(\alpha, \beta, \cdot)$ are graphed for various choices of $(\alpha, \beta)$ sampled from a horizontal line $(\beta=25)$ in regions $R(I), R(I I), R(I I I)$, and their boundaries; see Figure 2.

\section{Monotony and CRitical values}

It is easy to check that $\tau_{+}(\alpha, \beta, \cdot)$ is strictly decreasing on the interval $[-\pi / 2, \pi / 2]$ and $\tau_{-}(\alpha, \beta, \cdot)$ is strictly increasing on $[-\pi / 2, \pi / 2]$. 


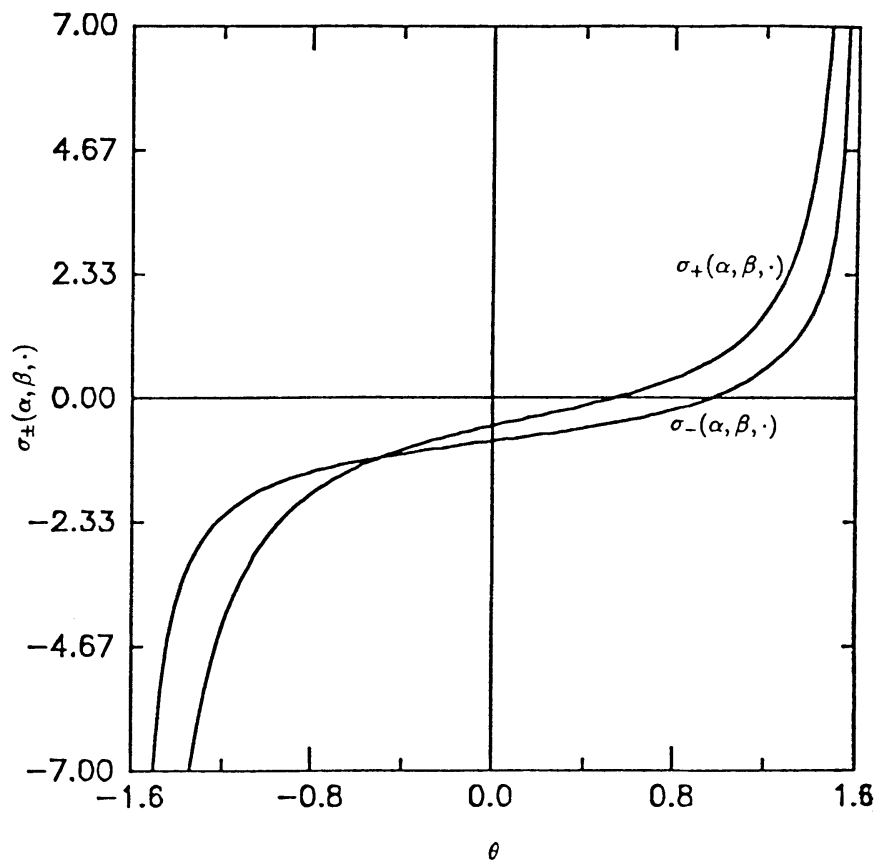

Figure 3. $\sigma_{+}(\alpha, \beta, \cdot)$ and $\sigma_{-}(\alpha, \beta, \cdot)$ for $(\alpha, \beta)=$ $(2.25,25) \in R(I)$

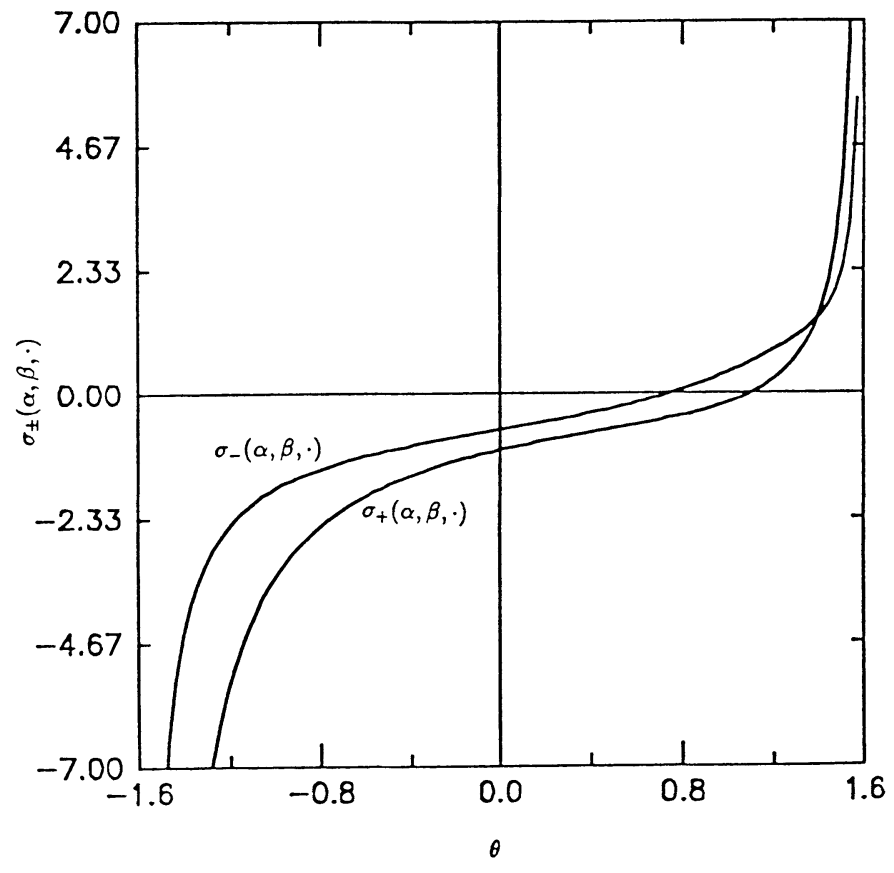

Figure 4. $\sigma_{+}(\alpha, \beta, \cdot)$ and $\sigma_{-}(\alpha, \beta, \cdot)$ for $(\alpha, \beta)=$ $(2.51,25) \in R(I)$ 


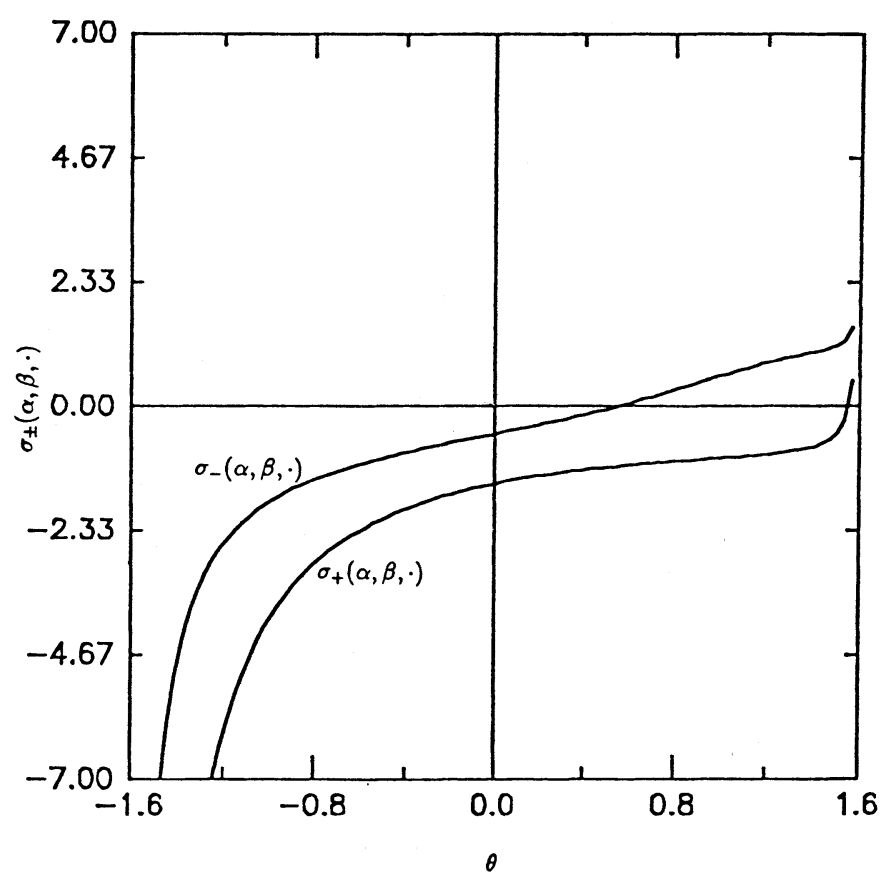

Figure 5. $\sigma_{+}(\alpha, \beta, \cdot)$ and $\sigma_{-}(\alpha, \beta, \cdot)$ for $(\alpha, \beta)=$ $(2.75,25) \in R(I)$

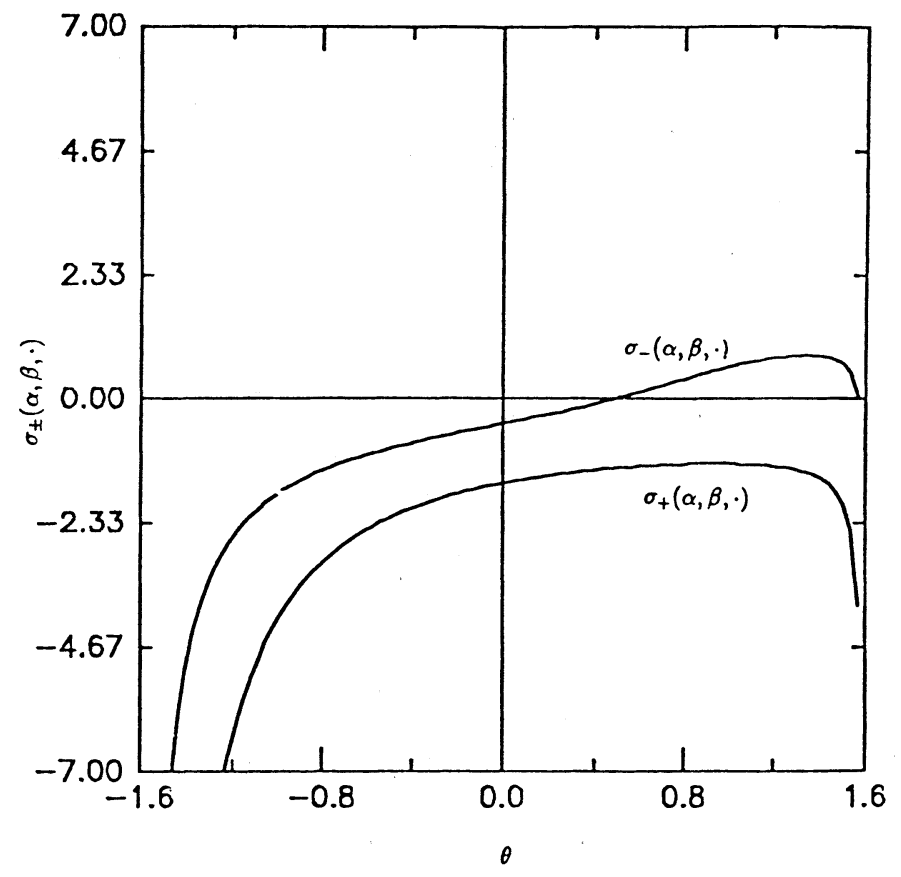

Figure 6. $\sigma_{+}(\alpha, \beta, \cdot)$ and $\sigma_{-}(\alpha, \beta, \cdot)$ for $(\alpha, \beta)=$ $(2.83,25) \in R(I I)$ 


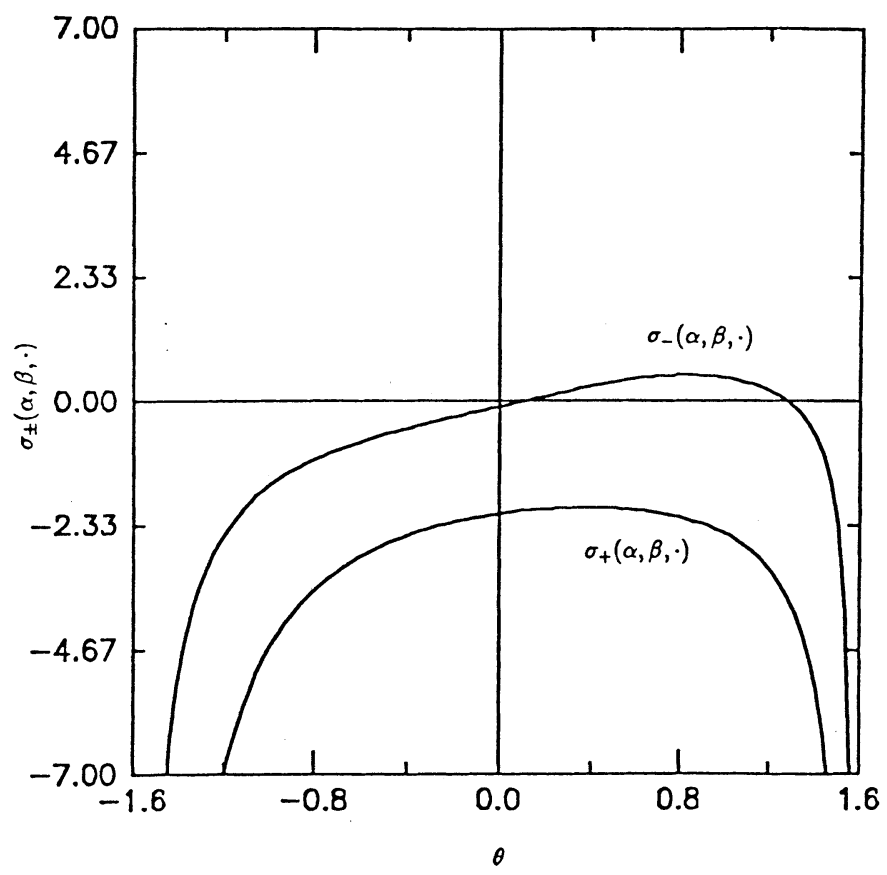

Figure 7. $\sigma_{+}(\alpha, \beta, \cdot)$ and $\sigma_{-}(\alpha, \beta, \cdot)$ for $(\alpha, \beta)=$ $(3.36,25) \in R(I I)$

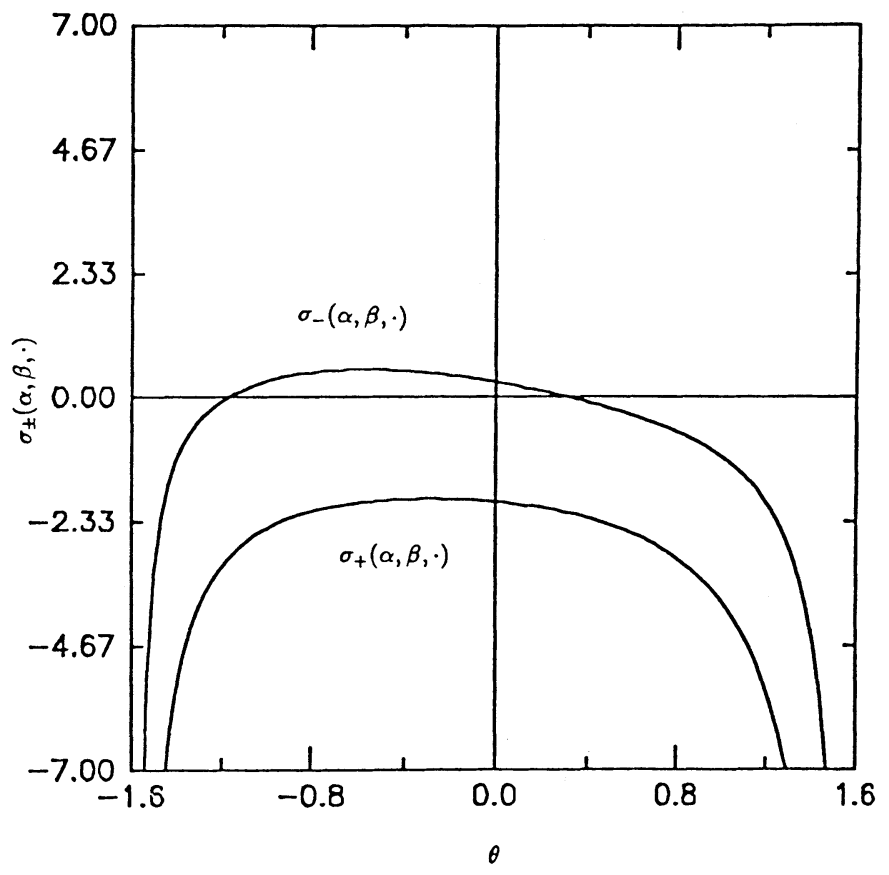

Figure 8. $\sigma_{+}(\alpha, \beta, \cdot)$ and $\sigma_{-}(\alpha, \beta, \cdot)$ for $(\alpha, \beta)=$ $(5.44,25) \in R(I I)$ 


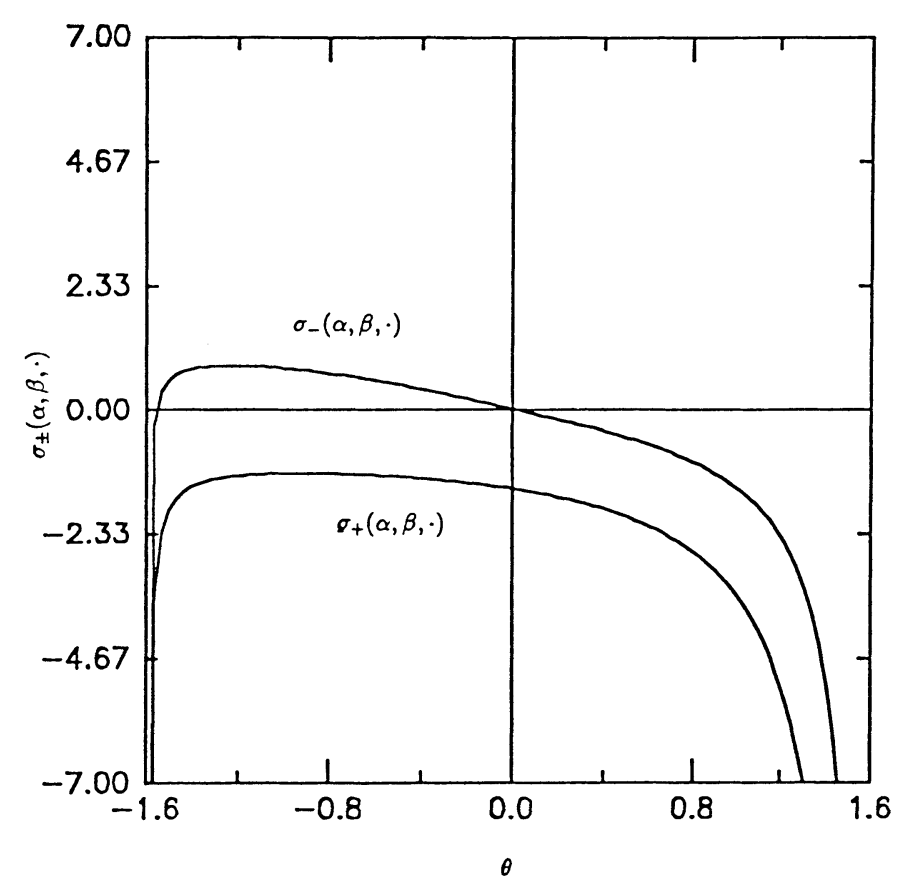

Figure 9. $\sigma_{+}(\alpha, \beta, \cdot)$ and $\sigma_{-}(\alpha, \beta, \cdot)$ for $(\alpha, \beta)=$ $(6.17,25) \in R(I I)$

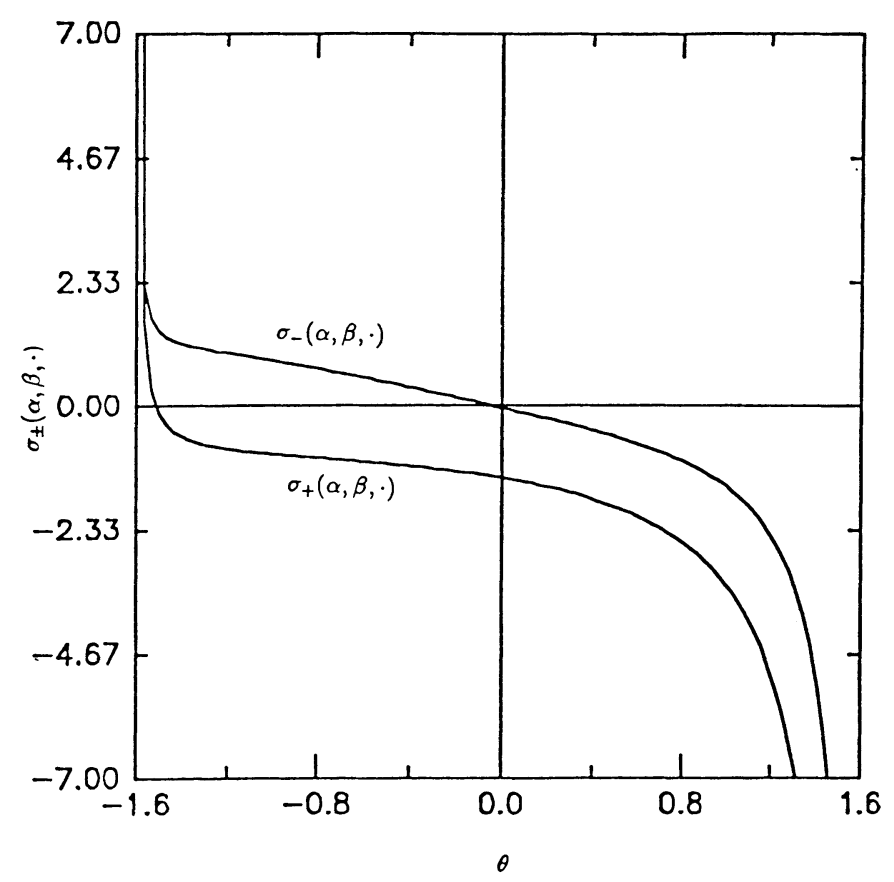

Figure 10. $\sigma_{+}(\alpha, \beta, \cdot)$ and $\sigma_{-}(\alpha, \beta, \cdot)$ for $(\alpha, \beta)=$ $(6.33,25) \in R(I I I)$ 


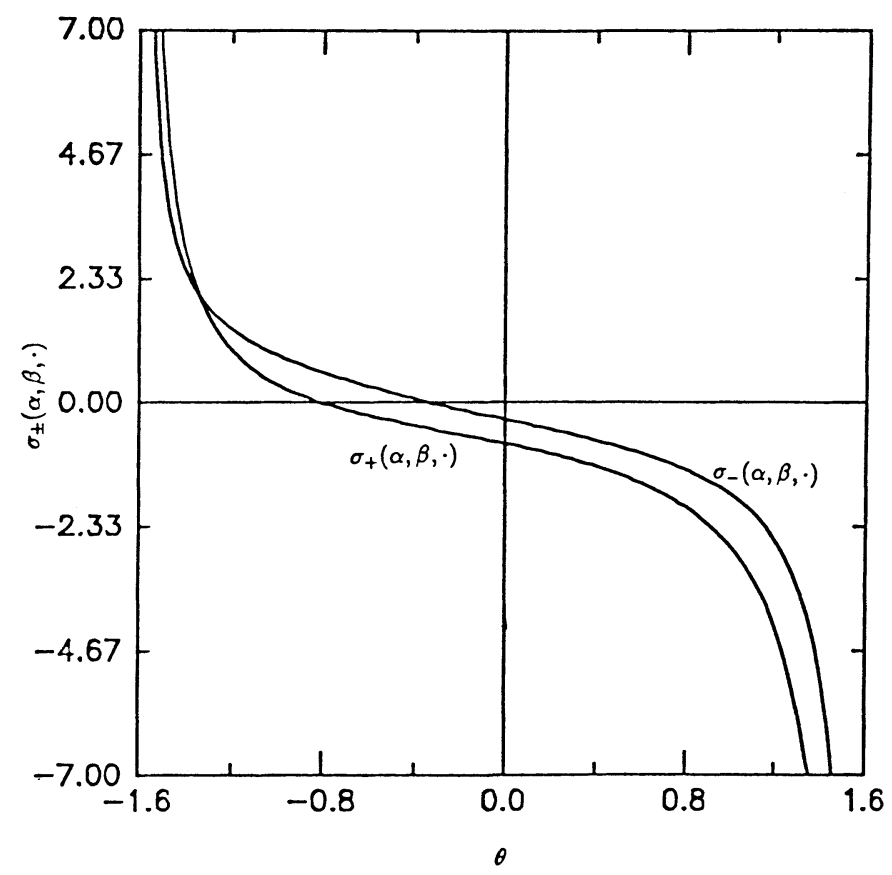

Figure 11. $\sigma_{+}(\alpha, \beta, \cdot)$ and $\sigma_{-}(\alpha, \beta, \cdot)$ for $(\alpha, \beta)=$ $(7.11,25) \in R(I I I)$

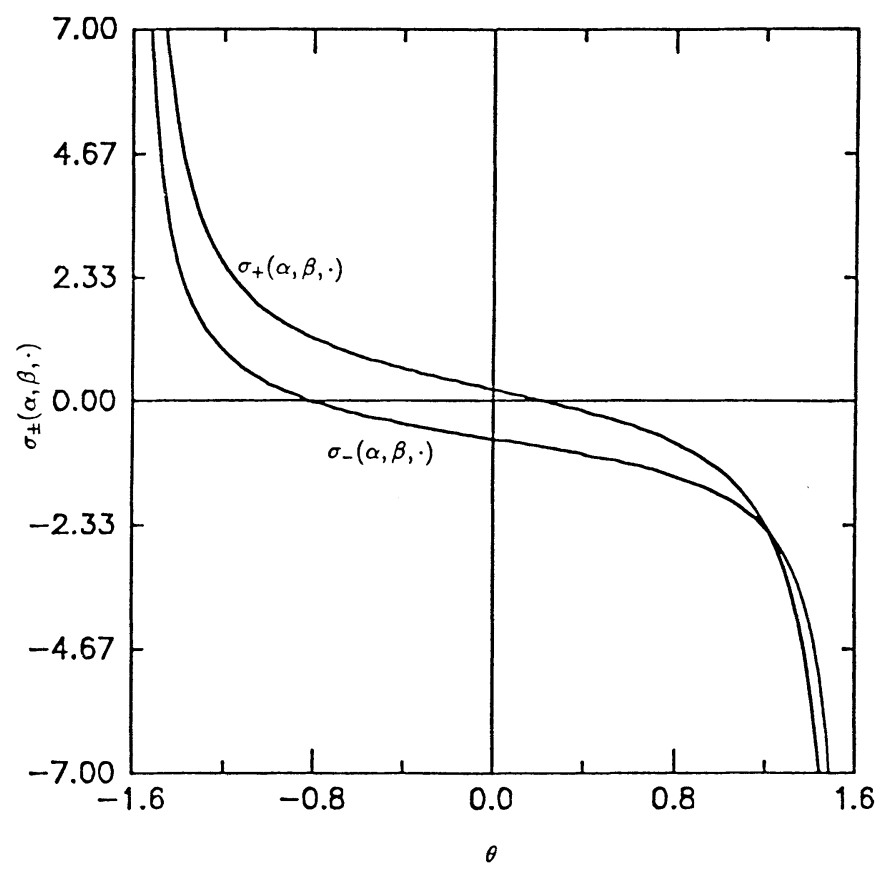

Figure 12. $\sigma_{+}(\alpha, \beta, \cdot)$ and $\sigma_{-}(\alpha, \beta, \cdot)$ for $(\alpha, \beta)=$ $(8.51,25) \in R(I I I)$ 


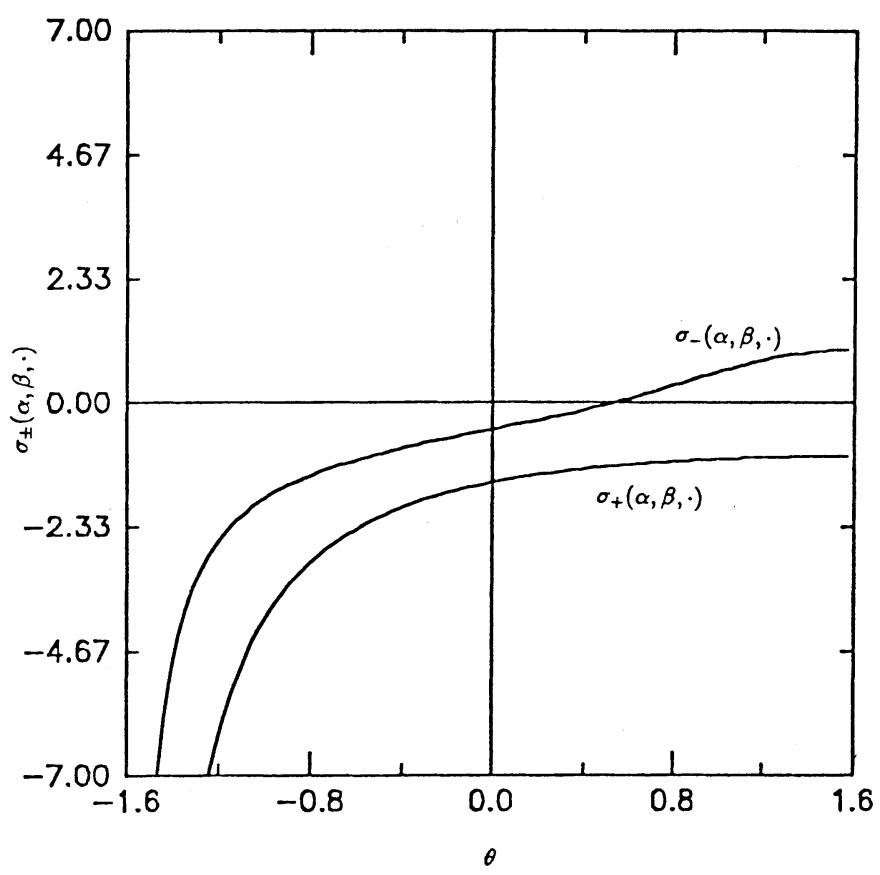

FigURE 13. $\sigma_{ \pm}(\alpha, \beta, \cdot)$ for $(\alpha, \beta)=(2.78,25) \in$ $\partial R(I) \cap \partial R(I I)$

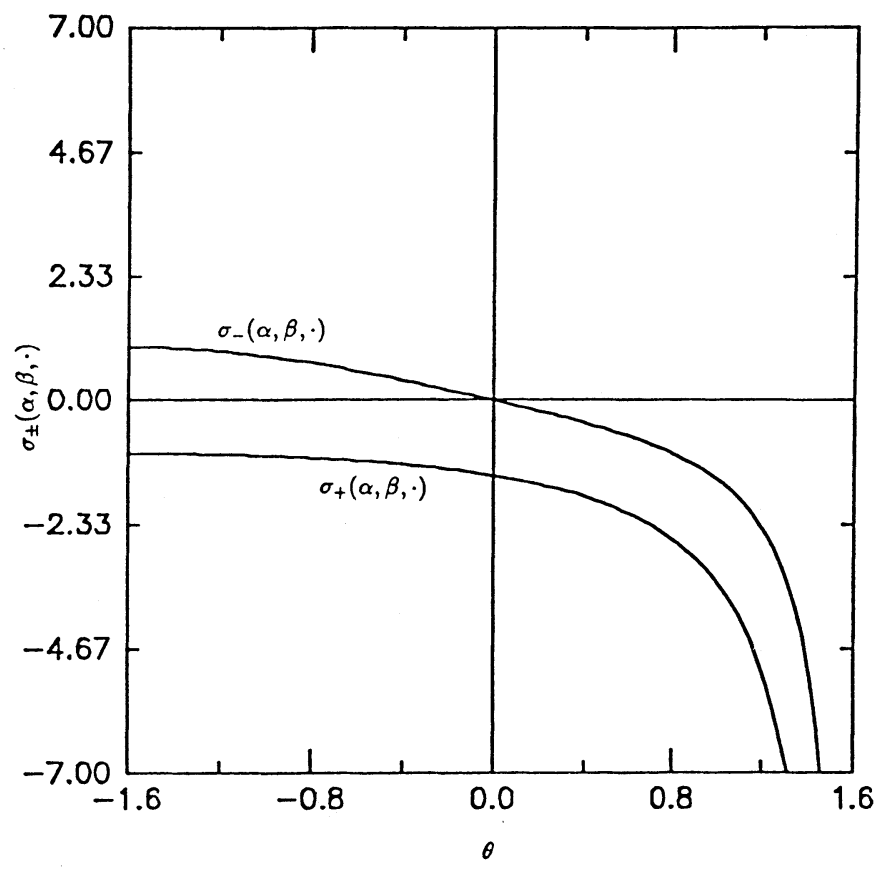

Figure 14. $\sigma_{ \pm}(\alpha, \beta, \cdot)$ for $(\alpha, \beta)=(6.25,25) \in$ $\partial R(I I) \cap \partial R(I I I)$ 
Differentiating $\sigma_{+}$and $\sigma_{-}$with respect to $\theta$ and using the relationship $\dot{u}=v$ gives

and

$$
\sigma_{+}^{\prime}(\alpha, \beta, \theta)=\left(1 / \cos ^{2} \theta\right) g_{+}(\alpha, \beta, \theta)
$$

$$
\sigma_{-}^{\prime}(\alpha, \beta, \theta)=\left(1 / \cos ^{2} \theta\right) g_{-}(\alpha, \beta, \theta)
$$

where $g_{ \pm}: Q^{+} \times[-\pi / 2, \pi / 2] \rightarrow \mathbf{R}$ are the continuous functions

$$
\begin{aligned}
g_{+}(\alpha, \beta, \theta) \stackrel{\text { def }}{=} & v\left[\tau_{+}(\alpha, \beta, \theta) ;\left(\begin{array}{c}
0 \\
-1
\end{array}\right)\right] \cos \theta \\
& -\sqrt{\beta} u\left[\tau_{+}(\alpha, \beta, \theta) ;\left(\begin{array}{c}
0 \\
-1
\end{array}\right)\right] \sin \theta,
\end{aligned}
$$

and

$$
\begin{aligned}
g_{-}(\alpha, \beta, \theta) \stackrel{\text { def }}{=} & v\left[\tau_{-}(\alpha, \beta, \theta) ;\left(\begin{array}{l}
0 \\
1
\end{array}\right)\right] \cos \theta \\
& +\sqrt{\alpha} u\left[\tau_{-}(\alpha, \beta, \theta) ;\left(\begin{array}{l}
0 \\
1
\end{array}\right)\right] \sin \theta .
\end{aligned}
$$

The zeros of $g_{ \pm}(\alpha, \beta, \cdot)$ in the open interval $(-\pi / 2, \pi / 2)$ then correspond to critical points of $\sigma_{ \pm}(\alpha, \beta, \cdot)$. In particular, the location of $(\alpha, \beta)$ will determine whether or not $g_{ \pm}(\alpha, \beta, \cdot)$ has a zero in $(-\pi / 2, \pi / 2)$, i.e., whether or not $\sigma_{ \pm}(\alpha, \beta, \cdot)$ has critical points in $(-\pi / 2, \pi / 2)$. The next lemma will be needed in determining such "critical" $(\alpha, \beta)$.

Lemma 1. $g_{ \pm}(\alpha, \beta, \cdot):[-\pi / 2, \pi / 2] \rightarrow \mathbf{R}$ are monotone functions.

Proof. To check this first fix $(\alpha, \beta) \in Q^{+}$and then differentiate $g_{+}$with respect to $\theta$ to get

$$
g_{+}^{\prime}(\alpha, \beta, \theta)=-\frac{\cos \theta}{\sqrt{\beta}}\left\{\dot{v}\left[\tau_{+}(\alpha, \beta, \theta) ;\left(\begin{array}{c}
0 \\
-1
\end{array}\right)\right]+\beta u\left[\tau(\alpha, \beta, \theta) ;\left(\begin{array}{c}
0 \\
-1
\end{array}\right)\right]\right\} .
$$

To compute the sign of $g_{+}^{\prime}$ we note that either

$$
u\left[\tau_{+}(\alpha, \beta, \theta) ;\left(\begin{array}{c}
0 \\
-1
\end{array}\right)\right] \geq 0 \text { or } u\left[\tau_{+}(\alpha, \beta, \theta) ;\left(\begin{array}{c}
0 \\
-1
\end{array}\right)\right]<0 .
$$

If it is the case that $u\left[\tau_{+}(\alpha, \beta, \theta) ;\left(\begin{array}{c}0 \\ -1\end{array}\right)\right] \geq 0$ then $\dot{v}=-\beta u$ and hence

$$
g_{+}^{\prime}(\alpha, \beta, \theta)=0 \text {. }
$$

If, on the other hand, $u\left[\tau_{+}(\alpha, \beta, \theta) ;\left(\begin{array}{c}0 \\ -1\end{array}\right)\right]<0$ then $\dot{v}=-\alpha u$ and so

$$
\begin{aligned}
g_{+}^{\prime}(\alpha, \beta, \theta)= & -\frac{\cos \theta}{\sqrt{\beta}}\left\{-\alpha u\left[\tau_{+}(\alpha, \beta, \theta) ;\left(\begin{array}{c}
0 \\
-1
\end{array}\right)\right]\right. \\
& \left.+\beta u\left[\tau_{+}(\alpha, \beta, \theta) ;\left(\begin{array}{c}
0 \\
-1
\end{array}\right)\right]\right\} \\
& =-\frac{\cos \theta}{\sqrt{\beta}} u\left[\tau_{+}(\alpha, \beta, \theta) ;\left(\begin{array}{c}
0 \\
-1
\end{array}\right)\right](\beta-\alpha) \\
& >0
\end{aligned}
$$


Thus, $g_{+}^{\prime}(\alpha, \beta, \theta) \geq 0$ whenever $\theta \in[-\pi / 2, \pi / 2]$, which, of course, shows $g_{+}(\alpha, \beta, \theta)$ to be nondecreasing. Moreover, $g_{+}(\alpha, \beta, \cdot)$ is strictly increasing whenever $\theta \in(-\pi / 2, \pi / 2)$ is such that

$$
u\left[\tau_{+}(\alpha, \beta, \theta) ;\left(\begin{array}{c}
0 \\
-1
\end{array}\right)\right]<0 .
$$

The situation with $g_{-}(\alpha, \beta, \cdot)$ is much the same. Differentiation with respect to $\theta$ shows that

$$
g_{-}^{\prime}(\alpha, \beta, \theta)=\frac{\cos \theta}{\sqrt{\alpha}}\left\{\dot{v}\left[\tau_{-}(\alpha, \beta, \theta) ;\left(\begin{array}{l}
0 \\
1
\end{array}\right)\right]+\alpha u\left[\tau_{-}(\alpha, \beta, \theta) ;\left(\begin{array}{l}
0 \\
1
\end{array}\right)\right]\right\} .
$$

Hence,

$$
g_{-}^{\prime}(\alpha, \beta, \theta)=0 \quad \text { if } u\left[\tau_{-}(\alpha, \beta, \theta) ;\left(\begin{array}{l}
0 \\
1
\end{array}\right)\right] \leq 0,
$$

and

$$
g_{-}^{\prime}(\alpha, \beta, \theta)=-\frac{\cos \theta}{\sqrt{\alpha}}(\beta-\alpha) u\left[\tau_{-}(\alpha, \beta, \theta) ;\left(\begin{array}{l}
0 \\
1
\end{array}\right)\right]
$$

whenever

$$
u\left[\tau_{-}(\alpha, \beta, \theta) ;\left(\begin{array}{l}
0 \\
1
\end{array}\right)\right]>0 \text {. }
$$

Therefore, $g_{-}^{\prime} \leq 0$ for $\theta \in[-\pi / 2, \pi / 2]$. One concludes that $g_{-}$is nonincreasing on $[-\pi / 2, \pi / 2]$ and strictly decreasing when $\theta \in(-\pi / 2, \pi / 2)$ is such that $u\left[\tau_{-}(\alpha, \beta, \theta) ;\left(\begin{array}{l}0 \\ 1\end{array}\right)\right]>0$.

6.1. In what follows we examine the behavior of the graphs of $\sigma_{+}$and $\sigma_{-}$for particular choices of the parameters $\alpha$ and $\beta$ taken from regions $R(I), R(I I)$. $R(I I I)$, and their boundaries. We suppose that a nonnegative integer $k$ has been fixed so that these regions are well defined.

If $(\alpha, \beta) \in R(I)$ then by (32) we obtain the following estimates:

$$
\begin{aligned}
\tau_{+}\left(\alpha, \beta,-\frac{\pi}{2}\right) & =\pi\left[1-\left(\frac{k}{\sqrt{\alpha}}+\frac{k}{\sqrt{\beta}}\right)\right]>0, \\
\tau_{+}\left(\alpha, \beta,-\frac{\pi}{2}\right) & =\pi\left[1-\left(\frac{k}{\sqrt{\alpha}}+\frac{k+1}{\sqrt{\beta}}\right)\right]+\frac{\pi}{\sqrt{\beta}}<\frac{\pi}{\sqrt{\beta}}<\frac{\pi}{\sqrt{\alpha}}, \\
\tau_{+}\left(\alpha, \beta, \frac{\pi}{2}\right) & =\pi\left[1-\left(\frac{k}{\sqrt{\alpha}}+\frac{k+1}{\sqrt{\beta}}\right)\right]<0, \\
\tau_{+}\left(\alpha, \beta, \frac{\pi}{2}\right) & =\pi\left[1-\left(\frac{k}{\sqrt{\alpha}}+\frac{k}{\sqrt{\beta}}\right)\right]-\frac{\pi}{\sqrt{\beta}}>-\frac{\pi}{\sqrt{\beta}} .
\end{aligned}
$$

Combining these estimates yields

$$
-\pi / \sqrt{\beta}<\tau_{+}(\alpha, \beta, \pi / 2)<0<\tau_{+}(\alpha, \beta,-\pi / 2)<\pi / \sqrt{\alpha}
$$

and hence

$$
u\left[\tau_{+}\left(\alpha, \beta,-\frac{\pi}{2}\right) ;\left(\begin{array}{c}
0 \\
-1
\end{array}\right)\right]<0, \quad u\left[\tau_{+}\left(\alpha, \beta, \frac{\pi}{2}\right) ;\left(\begin{array}{c}
0 \\
-1
\end{array}\right)\right]>0 .
$$


Similarly, one finds that

$$
-\pi / \sqrt{\alpha}<\tau_{-}(\alpha, \beta,-\pi / 2)<0<\tau_{-}(\alpha, \beta, \pi / 2)<\pi / \sqrt{\beta} ;
$$

hence

$$
u\left[\tau_{-}\left(\alpha, \beta,-\frac{\pi}{2}\right) ;\left(\begin{array}{l}
0 \\
1
\end{array}\right)\right]<0, \quad u\left[\tau_{-}\left(\alpha, \beta, \frac{\pi}{2}\right) ;\left(\begin{array}{l}
0 \\
1
\end{array}\right)\right]>0 .
$$

In view of $(38)$ then

$$
g_{+}\left(\alpha, \beta, \frac{\pi}{2}\right)=-\sqrt{\beta} u\left[\tau_{+}\left(\alpha, \beta, \frac{\pi}{2}\right) ;\left(\begin{array}{l}
0 \\
1
\end{array}\right)\right]<0 .
$$

From Lemma $1, g_{+}(\alpha, \beta, \cdot):[-\pi / 2, \pi / 2] \rightarrow \mathbf{R}$ is nondecreasing and hence

$$
g_{+}(\alpha, \beta, \theta)<0 \text { for } \theta \in[-\pi / 2, \pi / 2] .
$$

Evidently then,

$$
\sigma_{+}^{\prime}(\alpha, \beta, \theta)>0 \text { for } \theta \in(-\pi / 2, \pi / 2)
$$

and it follows that

$$
\sigma_{+}(\alpha, \beta, \cdot):(-\pi / 2, \pi / 2) \rightarrow \mathbf{R}
$$

is a strictly increasing function.

By a similar argument we have that

$$
\sigma_{-}(\alpha, \beta, \cdot):(-\pi / 2, \pi / 2) \rightarrow \mathbf{R}
$$

is also a strictly increasing function.

By (40) and (41)

$$
\sigma_{ \pm}(\alpha, \beta, \theta) \rightarrow\left\{\begin{array}{l}
-\infty \quad \text { as } \theta \rightarrow-\pi / 2 \\
\infty \quad \text { as } \theta \rightarrow \pi / 2
\end{array}\right.
$$

and hence conclude that $\sigma_{ \pm}(\alpha, \beta, \cdot):(-\pi / 2, \pi / 2) \rightarrow \mathbf{R}$ are homeomorphisms onto $\mathbf{R}$. This shows that problems (3) and (5) have unique solutions for each $\sigma \in \mathbf{R}$.

6.2. Now suppose that

$$
(\alpha, \beta) \in R(I I)=\left\{(\alpha, \beta) \mid 1-\left(\frac{k}{\sqrt{\alpha}}+\frac{k+1}{\sqrt{\beta}}\right)>0 ; 1-\left(\frac{k+1}{\sqrt{\alpha}}+\frac{k}{\sqrt{\beta}}\right)<0\right\}
$$

for some $k=0,1,2, \ldots$ From (32)

$$
\begin{aligned}
\tau_{+}\left(\alpha, \beta,-\frac{\pi}{2}\right) & =\pi\left[1-\left(\frac{k}{\sqrt{\alpha}}+\frac{k}{\sqrt{\beta}}\right)\right] \\
& =\pi\left[1-\left(\frac{k+1}{\sqrt{\alpha}}+\frac{k}{\sqrt{\beta}}\right)\right]+\frac{\pi}{\sqrt{\alpha}}<\frac{\pi}{\sqrt{\alpha}}
\end{aligned}
$$

and

$$
\tau_{+}\left(\alpha, \beta, \frac{\pi}{2}\right)=\pi\left[1-\left(\frac{k}{\sqrt{\alpha}}+\frac{k}{\sqrt{\beta}}\right)\right]-\frac{\pi}{\sqrt{\beta}}>0 .
$$


Therefore,

$$
0<\tau_{+}\left(\alpha, \beta, \frac{\pi}{2}\right)<\tau_{+}(\alpha, \beta, \theta)<\tau_{+}\left(\alpha, \beta,-\frac{\pi}{2}\right)<\frac{\pi}{\sqrt{\alpha}}
$$

for $\theta \in(-\pi / 2, \pi / 2)$ and so

$$
u\left[\tau_{+}(\alpha, \beta, \theta) ;\left(\begin{array}{c}
0 \\
-1
\end{array}\right)\right]<0 \text { for } \theta \in[-\pi / 2, \pi / 2] .
$$

It follows from (36) that

$$
\sigma_{+}(\alpha, \beta, \theta)<0 \text { for } \theta \in(-\pi / 2, \pi / 2)
$$

with

$$
\lim _{|\theta| \rightarrow \pi / 2} \sigma_{+}(\alpha, \beta, \theta)=-\infty
$$

Since

$$
g_{+}\left(\alpha, \beta,-\frac{\pi}{2}\right)=\sqrt{\beta} u\left[\tau_{+}\left(\alpha, \beta,-\frac{\pi}{2}\right) ;\left(\begin{array}{c}
0 \\
-1
\end{array}\right)\right]<0
$$

and

$$
g_{+}\left(\alpha, \beta, \frac{\pi}{2}\right)=-\sqrt{\beta} u\left[\tau_{+}\left(\alpha, \beta, \frac{\pi}{2}\right) ;\left(\begin{array}{c}
0 \\
-1
\end{array}\right)\right]>0
$$

recalling that $g_{+}(\alpha, \beta, \cdot)$ is strictly increasing in $\theta$ whenever $\theta$ is such that $u\left[\tau_{+}(\alpha, \beta, \theta) ;\left(\begin{array}{c}0 \\ -1\end{array}\right)\right]<0$, it follows that $g_{+}(\alpha, \beta, \cdot)$ has precisely one zero in the open interval $(-\pi / 2, \pi / 2)$. Consequently, $\sigma_{+}(\alpha, \beta, \cdot)$ has exactly one critical point, say $\theta_{0}$, in $(-\pi / 2, \pi / 2)$ and at this point $\sigma_{+}\left(\alpha, \beta, \theta_{0}\right)<0$.

Moreover,

$$
\sigma_{+}^{\prime}(\alpha, \beta, \theta)=-\left(1 / \cos ^{2} \theta\right) g_{+}(\alpha, \beta, \theta)
$$

shows that

$$
\sigma_{+}^{\prime}(\alpha, \beta, \theta)>0, \quad \theta \in\left(-\pi / 2, \theta_{0}\right)
$$

and

$$
\sigma_{+}^{\prime}(\alpha, \beta, \theta)<0, \quad \theta \in\left(\theta_{0}, \pi / 2\right) .
$$

This indicates that $\sigma_{+}(\alpha, \beta, \cdot)$ is strictly increasing when $\theta<\theta_{0}$ and strictly decreasing when $\theta>\theta_{0}$. Put

$$
M_{+}(\alpha, \beta) \stackrel{\text { def }}{=} \max _{-\pi / 2<\theta<\pi / 2} \sigma_{+}(\alpha, \beta, \theta)
$$

then $M_{+}<0$ and, in view of (43), it is evident that problem (3) has no solution if $\sigma>M_{+}(\alpha, \beta)$, a unique solution if $\sigma=M_{+}(\alpha, \beta)$, and exactly two distinct solutions if $\sigma<M_{+}(\alpha, \beta)$. This is the familiar Ambrosetti-Prodi situation.

The argument for $\sigma_{-}$is similar. That is, $(\alpha, \beta) \in R(I I)$ and equation (33) give the following chain of estimates:

$$
-\frac{\pi}{\sqrt{\alpha}}<\tau_{-}\left(\alpha, \beta,-\frac{\pi}{2}\right)<0<\frac{\pi}{\sqrt{\beta}}<\tau_{-}\left(\alpha, \beta,-\frac{\pi}{2}\right)<\frac{\pi}{\sqrt{\alpha}}+\frac{\pi}{\sqrt{\beta}} .
$$


So

and from (37)

$$
u\left[\tau\left(\alpha, \beta, \pm \frac{\pi}{2}\right) ;\left(\begin{array}{l}
0 \\
1
\end{array}\right)\right]<0
$$

$$
\lim _{|\theta| \rightarrow \pm \pi / 2} \sigma_{-}(\alpha, \beta, \theta)=-\infty .
$$

Since $\tau_{-}(\alpha, \beta, \cdot):[-\pi / 2, \pi / 2] \rightarrow \mathbf{R}$ is strictly increasing, from (44) there is a nontrivial subinterval $\left[\theta_{1}, \theta_{2}\right] \subset(-\pi / 2, \pi / 2)$ such that $\theta \in\left[\theta_{1}, \theta_{2}\right]$ implies $0 \leq \tau_{-}(\alpha, \beta, \theta) \leq \pi / \sqrt{\beta}$ and hence

$$
u\left[\tau_{-}(\alpha, \beta, \theta) ;\left(\begin{array}{l}
0 \\
1
\end{array}\right)\right]>0 \text { on }\left(\theta_{1}, \theta_{2}\right) \text {. }
$$

Using (39) gives the estimates

$$
g_{-}\left(\alpha, \beta,-\frac{\pi}{2}\right)=-\sqrt{\alpha} u\left[\tau_{-}\left(\alpha, \beta,-\frac{\pi}{2}\right) ;\left(\begin{array}{l}
0 \\
1
\end{array}\right)\right]>0
$$

and

$$
g_{-}\left(\alpha, \beta,-\frac{\pi}{2}\right)=\sqrt{\alpha} u\left[\tau_{-}\left(\alpha, \beta,-\frac{\pi}{2}\right) ;\left(\begin{array}{l}
0 \\
1
\end{array}\right)\right]<0 .
$$

Since $g_{-}^{\prime}(\alpha, \beta, \theta)=0$ for $\theta \in(-\pi / 2, \pi / 2) \backslash\left[\theta_{1}, \theta_{2}\right]$ (cf. Lemma 1) it follows that

$$
0<g_{-}(\alpha, \beta,-\pi / 2) \equiv g_{-}(\alpha, \beta, \theta), \quad \theta \in\left[-\pi / 2, \theta_{1}\right),
$$

and

$$
0>g_{-}(\alpha, \beta, \pi / 2) \equiv g_{-}(\alpha, \beta, \theta), \quad \theta \in\left(\theta_{2}, \pi / 2\right],
$$

with $g_{-}(\alpha, \beta, \cdot)$ strictly decreasing on $\left(\theta_{1}, \theta_{2}\right)$. Let $\hat{\theta}_{0}$ be the unique point in $\left(\theta_{1}, \theta_{2}\right)$ such that $g_{-}\left(\alpha, \beta, \widehat{\theta}_{0}\right)=0$; then

$$
\begin{array}{cc}
g_{-}(\alpha, \beta, \theta)>0, & \theta \in\left[-\pi / 2, \widehat{\theta}_{0}\right), \\
g_{-}(\alpha, \beta, \theta)<0, & \theta \in\left(\widehat{\theta}_{0}, \pi / 2\right],
\end{array}
$$

and hence

$$
\sigma_{-}^{\prime}(\alpha, \beta, \theta)>0, \quad \theta \in\left(-\pi / 2, \widehat{\theta}_{0}\right),
$$

and

$$
\sigma_{-}^{\prime}(\alpha, \beta, \theta)<0, \quad \theta \in\left(\widehat{\theta}_{0}, \pi / 2\right)
$$

Define

$$
M_{-}(\alpha, \beta) \stackrel{\text { def }}{=} \max _{-\pi / 2<\theta<\pi / 2} \sigma_{-}(\alpha, \beta, \theta) .
$$

Note that $M_{-}(\alpha, \beta)>0$ and, as in the case for $\sigma_{+}$, one concludes that problem (5) has no solution if $\sigma>M_{-}(\alpha, \beta)$, a unique solution if $\sigma=M_{-}(\alpha, \beta)$, and exactly two solutions if $\sigma<M_{-}(\alpha, \beta)$.

There is a relationship between $M_{+}(\alpha, \beta)$ and $M_{-}(\alpha, \beta)$. In Theorem 3 it will be shown that $M_{-}(\alpha, \beta)=-1 / M_{+}(\alpha, \beta)$.

Figures $6,7,8$, and 9 contain graphs of $\sigma_{+}(\alpha, \beta, \cdot)$ and $\sigma_{-}(\alpha, \beta, \cdot)$ for $(\alpha, \beta)$ in region $R(I I)$ (see Figure 2) chosen from the line $\beta=25$. 
6.3. Fix $(\alpha, \beta)$ in $R(I I I)$, where we recall

$$
R(I I I)=\left\{(\alpha, \beta) \mid \alpha<\beta ; 1-\left(\frac{k+1}{\sqrt{\alpha}}+\frac{k}{\sqrt{\beta}}\right)>0 ; 1-\left(\frac{k+1}{\sqrt{\alpha}}+\frac{k+1}{\sqrt{\beta}}\right)<0\right\}
$$

(see Figure 2). The situation here is similar to the case when $(\alpha, \beta) \in R(I)$. Using (32) and estimating yields,

$$
0<\tau_{+}\left(\alpha, \beta, \frac{\pi}{2}\right)<\frac{\pi}{\sqrt{\alpha}}<\tau_{+}\left(\alpha, \beta,-\frac{\pi}{2}\right)<\frac{\pi}{\sqrt{\alpha}}+\frac{\pi}{\sqrt{\beta}} .
$$

Similar use of (33) leads to inequalities

$$
0<\tau_{-}\left(\alpha, \beta,-\frac{\pi}{2}\right)<\frac{\pi}{\sqrt{\beta}}<\tau_{-}\left(\alpha, \beta, \frac{\pi}{2}\right)<\frac{\pi}{\sqrt{\alpha}}+\frac{\pi}{\sqrt{\beta}} .
$$

Evidently then,

$$
\begin{gathered}
u\left[\tau_{+}\left(\alpha, \beta, \frac{\pi}{2}\right) ;\left(\begin{array}{c}
0 \\
-1
\end{array}\right)\right]<0, \\
u\left[\tau_{+}\left(\alpha, \beta,-\frac{\pi}{2}\right) ;\left(\begin{array}{c}
0 \\
-1
\end{array}\right)\right]>0, \\
u\left[\tau_{-}\left(\alpha, \beta, \frac{\pi}{2}\right) ;\left(\begin{array}{c}
0 \\
1
\end{array}\right)\right]<0, \\
u\left[\tau_{-}\left(\alpha, \beta,-\frac{\pi}{2}\right) ;\left(\begin{array}{l}
0 \\
1
\end{array}\right)\right]>0,
\end{gathered}
$$

and, from (36) and (37), it follows that

$$
\sigma_{ \pm}(\alpha, \beta, \theta) \rightarrow \begin{cases}\infty & \text { as } \theta \rightarrow-\pi / 2 \\ -\infty & \text { as } \theta \rightarrow \pi / 2 .\end{cases}
$$

The estimates

$$
\begin{aligned}
& g_{+}\left(\alpha, \beta,-\frac{\pi}{2}\right)=\sqrt{\beta} u\left[\tau_{+}\left(\alpha, \beta,-\frac{\pi}{2}\right) ;\left(\begin{array}{c}
0 \\
-1
\end{array}\right)\right]>0, \\
& g_{-}\left(\alpha, \beta,-\frac{\pi}{2}\right)=-\sqrt{\alpha} u\left[\tau_{-}\left(\alpha, \beta,-\frac{\pi}{2}\right) ;\left(\begin{array}{c}
0 \\
1
\end{array}\right)\right]<0,
\end{aligned}
$$

and fact that the $g_{ \pm}(\alpha, \beta, \cdot)$ are monotone (see Lemma 1) shows that

$$
g_{+}(\alpha, \beta, \theta)>0, \quad \theta \in[-\pi / 2, \pi / 2],
$$

and

$$
g_{-}(\alpha, \beta, \theta)<0, \quad \theta \in[-\pi / 2, \pi / 2] \text {. }
$$

Hence

$$
\sigma_{ \pm}^{\prime}(\alpha, \beta, \theta)<0 \text { for } \theta \in(-\pi / 2, \pi / 2)
$$

i.e., both $\sigma_{ \pm}(\alpha, \beta, \cdot)$ are strictly decreasing on the interval $(-\pi / 2, \pi / 2)$.

Finally, as in the case when $(\alpha, \beta) \in R(I),(45)$ is enough to conclude that

$$
\sigma_{ \pm}(\alpha, \beta, \cdot):(-\pi / 2, \pi / 2) \rightarrow \mathbf{R}
$$


is a homeomorphism onto $\mathbf{R}$ whenever $(\alpha, \beta) \in R(I I I)$. Problems (3) and (5) then each have unique solutions for each $\sigma \in \mathbf{R}$.

Graphs of $\sigma_{+}$and $\sigma_{-}$, when $(\alpha, \beta) \in R(I I I)$ and $\beta=25$ are shown in Figures 10, 11, and 12 .

6.4. Consider the boundary situation $(\alpha, \beta) \in \partial R(I) \cap \partial R(I I)$ with $\alpha<\beta$; i.e., $(\alpha, \beta)$ is found on the curve

$$
k / \sqrt{\alpha}+(k+1) / \sqrt{\beta}=1 .
$$

From (32)

$$
\tau_{+}(\alpha, \beta, \theta)=\pi\left[1-\left(\frac{k}{\sqrt{\alpha}}+\frac{k}{\sqrt{\beta}}\right)\right]-\frac{\pi}{2 \sqrt{\beta}}-\frac{1}{\sqrt{\beta}} \theta=\frac{\pi}{2 \sqrt{\beta}}-\frac{1}{\sqrt{\beta}} \theta
$$

and it follows that

$$
0=\tau_{+}(\alpha, \beta, \pi / 2)<\tau_{+}(\alpha, \beta, \theta)<\tau_{+}(\alpha, \beta,-\pi / 2)=-\pi / \sqrt{\beta}
$$

for $\theta \in(-\pi / 2, \pi / 2)$. As before, this implies

$$
u\left[\tau_{+}(\alpha, \beta, \theta) ;\left(\begin{array}{c}
0 \\
-1
\end{array}\right)\right]<0, \quad \theta \in\left[-\frac{\pi}{2}, \frac{\pi}{2}\right)
$$

with

$$
u\left[\tau_{+}\left(\alpha, \beta, \frac{\pi}{2}\right) ;\left(\begin{array}{c}
0 \\
-1
\end{array}\right)\right]=0
$$

Recalling that

$$
\sigma_{+}(\alpha, \beta, \theta)=\sqrt{\beta} \frac{u\left[\tau_{+}(\alpha, \beta, \theta) ;\left(\begin{array}{c}
0 \\
-1
\end{array}\right)\right]}{\cos \theta},
$$

then

$$
\sigma_{+}(\alpha, \beta, \theta)<0, \quad \theta \in(-\pi / 2, \pi / 2)
$$

and

$$
\lim _{\theta \rightarrow-\pi / 2} \sigma_{+}(\alpha, \beta, \theta)=-\infty
$$

Using l'Hôpital's rule yields

$$
\begin{aligned}
\lim _{\theta \rightarrow \pi / 2} \sigma_{+}(\alpha, \beta, \theta) & =\lim _{\theta \rightarrow \pi / 2} \sqrt{\beta}\left\{\frac{\dot{u}\left[\tau_{+}(\alpha, \beta, \theta) ;\left(\begin{array}{c}
0 \\
-1
\end{array}\right)\right] \tau_{+}^{\prime}(\alpha, \beta, \theta)}{-\sin \theta}\right\} \\
& =v\left[\tau_{+}\left(\alpha, \beta, \frac{\pi}{2}\right) ;\left(\begin{array}{c}
0 \\
-1
\end{array}\right)\right]=v\left[0 ;\left(\begin{array}{c}
0 \\
-1
\end{array}\right)\right] \\
& =-1 .
\end{aligned}
$$

From $(46), g_{+}(\alpha, \beta, \cdot)$ is strictly increasing on $[-\pi / 2, \pi / 2)$ and since

$$
g_{+}\left(\alpha, \beta, \frac{\pi}{2}\right)=-\sqrt{\beta} u\left[\tau_{+}\left(\alpha, \beta, \frac{\pi}{2}\right) ;\left(\begin{array}{c}
0 \\
-1
\end{array}\right)\right]=0,
$$


then $g_{+}(\alpha, \beta, \theta)<0$ on $[-\pi / 2, \pi / 2)$. Hence,

$$
\sigma_{+}^{\prime}(\alpha, \beta, \theta)>0 \text { for } \theta \in(-\pi / 2, \pi / 2) \text {; }
$$

i.e., $\sigma_{+}(\alpha, \beta, \cdot)$ is strictly increasing up to its value of -1 , which it attains at $\theta=\pi / 2$. See Figure 5 for a graph of $\sigma_{+}(\alpha, \beta, \cdot)$. In this situation we may conclude that if $\sigma \geq-1$ problem (3) has no solution and if $\sigma<-1$ a unique solution.

The argument for $\sigma_{-}$is similar. It results in problem (5) having no solution if $\sigma \geq 1$ and a unique solution if $\sigma<1$. See Figure 13 for graphs of $\sigma_{+}$and $\sigma_{-}$.

6.5. Consider the boundary case when $\alpha<\beta$ and $(\alpha, \beta) \in \partial R(I I) \cap \partial R(I I I)$ for some $k=0,1,2, \ldots$ So

$$
(k+1) / \sqrt{\alpha}+k / \sqrt{\beta}=1
$$

and (32) becomes

$$
\begin{aligned}
\tau_{+}(\alpha, \beta, \theta) & =\pi\left[1-\left(\frac{k}{\sqrt{\alpha}}+\frac{k}{\sqrt{\beta}}\right)\right]-\frac{\pi}{2 \sqrt{\beta}}-\frac{1}{\sqrt{\beta}} \theta \\
& =\frac{\pi}{\sqrt{\alpha}}-\frac{\pi}{2 \sqrt{\beta}}-\frac{1}{\sqrt{\beta}} \theta .
\end{aligned}
$$

It follows that

$$
0<\tau_{+}(\alpha, \beta, \pi / 2)<\tau_{+}(\alpha, \beta, \theta)<\tau_{+}(\alpha, \beta,-\pi / 2)=\pi / \sqrt{\alpha},
$$

and thus

$$
u\left[\tau_{+}(\alpha, \beta, \theta) ;\left(\begin{array}{c}
0 \\
-1
\end{array}\right)\right]<0 \text { on }\left(-\frac{\pi}{2}, \frac{\pi}{2}\right]
$$

and

$$
u\left[\tau_{+}\left(\alpha, \beta,-\frac{\pi}{2}\right) ;\left(\begin{array}{c}
0 \\
-1
\end{array}\right)\right]=u\left[\frac{\pi}{\sqrt{\alpha}} ;\left(\begin{array}{c}
0 \\
-1
\end{array}\right)\right]=0 .
$$

This, in turn, shows that

$$
g_{+}\left(\alpha, \beta,-\frac{\pi}{2}\right)=\sqrt{\beta} u\left[\tau_{+}\left(\alpha, \beta,-\frac{\pi}{2}\right) ;\left(\begin{array}{c}
0 \\
-1
\end{array}\right)\right]=0 .
$$

For $\theta \in(-\pi / 2, \pi / 2],(47)$ and Lemma 1 show that $g_{+}$is increasing and hence

$$
g_{+}(\alpha, \beta, \theta)>0, \quad \theta \in(-\pi / 2, \pi / 2] .
$$

Consequently, $\sigma_{+}(\alpha, \beta, \cdot)$, is strictly decreasing on $(-\pi / 2, \pi / 2)$. Moreover,

$$
\sigma_{+}(\alpha, \beta, \theta) \rightarrow-\infty, \quad \text { as } \theta \rightarrow \pi / 2
$$

and applying l'Hôpital's rule once again yields

$$
\lim _{\theta \rightarrow-\pi / 2} \sigma_{+}(\alpha, \beta, \theta)=-1 \text {. }
$$

Thus problem (3) is solvable if and only if $\sigma<-1$, in which case it has a unique solution. 
The situation with $\sigma_{-}$is, again, much the same. One concludes that problem (5) is solvable if and only if $\sigma<1$, in which case the solution is unique.

Figure 14 contains a graph of $\sigma_{+}(\alpha, \beta, \cdot)$ and $\sigma_{-}(\alpha, \beta, \cdot)$ for $(\alpha, \beta) \in$ $\partial R(I I) \cap \partial R(I I I)$ and $\alpha<\beta$.

6.6. We consider the case when $(\alpha, \beta)$ belongs to a curve of the form

$$
k / \sqrt{\alpha}+k / \sqrt{\beta}=1
$$

for some $k=1,2,3, \ldots$ In this situation the solvability question of problem (2) may be answered directly, i.e., without appeal to any of the auxiliary problems (3), (4), or (5). That is, solutions of the system (7) have period $\pi / \sqrt{\alpha}+\pi / \sqrt{\beta}$, so if $\alpha$ and $\beta$ are chosen as above then

$$
k\{\pi / \sqrt{\alpha}+\pi / \sqrt{\beta}\}=\pi .
$$

Consequently, a solution starting at some point

$$
\left(\begin{array}{l}
u_{0} \\
v_{0}
\end{array}\right)
$$

in the phase plane will return there after time $\pi$. Hence, problem (2) is solvable if and only if $\sigma_{1}=\sigma_{2}$, in which case there are infinitely many solutions.

Remark. Up to this point we have determined the following: If $(\alpha, \beta) \in$ $R(I) \cup R(I I I)$, problems (3) and (5) have unique solutions for each $\sigma \in \mathbf{R}$. If $(\alpha, \beta) \in R(I I)$, there exists a negative real number $M_{+}=M_{+}(\alpha, \beta)$ such that problem (3) has either no solution, a unique solution, or exactly two solutions depending on whether $\sigma$ is greater than $M_{+}$, equal to $M_{+}$, or less than $M_{+}$, respectively. Similarly, there exists a positive real number $M_{-}=M_{-}(\alpha, \beta)$ such that problem (5) has either no solution, a unique solution, or exactly two solutions depending on whether $\sigma$ is greater than $M_{-}$, equal to $M_{-}$, or less than $M_{-}$, respectively.

If $(\alpha, \beta)$ belongs to the boundary $\partial R(I) \cap \partial R(I I)$.or $\partial R(I I) \cap \partial R(I I I)$ with $\alpha<\beta$, then (3) has no solution if $\sigma \geq-1$ and a unique solution if $\sigma<-1$ and problem (5) has no solution if $\sigma \geq 1$ and a unique solution if $\sigma<1$. Finally, if $(\alpha, \beta)$ belongs to a curve of the form

$$
k / \sqrt{\alpha}+k / \sqrt{\beta}=1,
$$

for some positive integer $k$, problems (3) and (5) are solvable if and only if $\sigma=1$ and $\sigma=-1$, respectively, in which case there are infinitely many solutions.

\section{THE CASE $u(0)=0$}

We have looked at two of our three reduced problems and are left with problem (4)

$$
\left\{\begin{array}{l}
u^{\prime \prime}+\alpha u^{-}+\beta u^{+}=0 \\
u(0)=0, \quad u(\pi)=\sigma
\end{array}\right.
$$


Results in this section parallel those of $\S 5$ but use somewhat cleaner arguments.

Before proceeding, note that if $\theta= \pm \pi / 2$ in (34) then

$$
u\left[\pi ;\left(\begin{array}{l}
0 \\
1
\end{array}\right)\right]=u\left[\tau_{+}\left(\alpha, \beta, \frac{\pi}{2}\right) ;\left(\begin{array}{c}
0 \\
-1
\end{array}\right)\right]
$$

and

$$
u\left[\pi ;\left(\begin{array}{c}
0 \\
-1
\end{array}\right)\right]=u\left[\tau_{+}\left(\alpha, \beta, \frac{\pi}{1}\right) ;\left(\begin{array}{c}
0 \\
-1
\end{array}\right)\right] .
$$

7.1. Fix $(\alpha, \beta)$ in $R(I)$ and recall from $\S 6.1$ the estimate (40)

$$
u\left[\tau_{+}\left(\alpha, \beta, \frac{\pi}{2}\right) ;\left(\begin{array}{c}
0 \\
-1
\end{array}\right)\right]<0 \text { and } u\left[\tau_{+}\left(\alpha, \beta, \frac{\pi}{2}\right) ;\left(\begin{array}{c}
0 \\
-1
\end{array}\right)\right]>0 \text {. }
$$

In particular then, using (49) and (50),

$$
u\left[\pi ;\left(\begin{array}{c}
0 \\
-1
\end{array}\right)\right]<0 \text { and } u\left[\pi ;\left(\begin{array}{l}
0 \\
1
\end{array}\right)\right]>0 .
$$

Thus, if $\sigma_{1}>0$ there is a unique positive real number $s_{1}=s_{1}\left(\sigma_{1}\right)$ (the correct shooting slope) such that

$$
s_{1} u\left[\pi ;\left(\begin{array}{l}
0 \\
1
\end{array}\right)\right]=\sigma_{1}
$$

Hence,

$$
w(t) \stackrel{\text { def }}{=} s_{1} u\left[t ;\left(\begin{array}{l}
0 \\
1
\end{array}\right)\right], \quad t \in[0, \pi],
$$

is a solution of (48) with $\sigma=\sigma_{1}>0$.

Conversely, if $w(t)$ is a solution of (4) with $\sigma=\sigma_{1}>0$, then by uniqueness

$$
\left(\begin{array}{c}
w(t) \\
\dot{w}(t)
\end{array}\right)=\left(\begin{array}{c}
u \\
v
\end{array}\right)\left[t ;\left(\begin{array}{c}
0 \\
\dot{w}(0)
\end{array}\right)\right], \quad t \in[0, \pi] .
$$

Note that $\dot{w}(0) \neq 0$ (otherwise $w \equiv 0$ ). Suppose that $\dot{w}(0)<0$; then by scaling

$$
\sigma_{1}=w(\pi)=u\left[\pi ;\left(\begin{array}{c}
0 \\
\dot{w}(0)
\end{array}\right)\right]=-\dot{w}(0) u\left[\pi ;\left(\begin{array}{c}
0 \\
-1
\end{array}\right)\right]<0,
$$

but this contradicts $\sigma_{1}>0$. Thus $\dot{w}(0)>0$ and

$$
w(t)=\dot{w}(0) u\left[t ;\left(\begin{array}{l}
0 \\
1
\end{array}\right)\right]
$$

with

$$
\sigma_{1}=w(\pi)=\dot{w}(0) u\left[\pi ;\left(\begin{array}{l}
0 \\
1
\end{array}\right)\right] .
$$

From (52) it follows then that $\dot{w}(0)=s_{1}$ and hence

$$
w(t)=s_{1} u\left[t ;\left(\begin{array}{l}
0 \\
1
\end{array}\right)\right], \quad t \in[0, \pi] .
$$

The above argument shows that problem (48) has a unique solution whenever $(\alpha, \beta) \in R(I)$ and $\sigma>0$. 
Again the situation when $\sigma=\sigma_{2}<0$ is essentially the same. Straightforward modifications to the above argument show (48) to have a unique solution whenever $(\alpha, \beta) \in R(I)$ and $\sigma<0$.

By the definition of $R(I),(48)$ with $\sigma=0$ has only the trivial solution and so we conclude that problem (48), when $(\alpha, \beta) \in R(I)$, is uniquely solvable for each $\sigma \in \mathbf{R}$.

7.2. Suppose now that $(\alpha, \beta)$ belongs to region $R(I I)$. From $\S 6.2$

$$
u\left[\tau_{+}(\alpha, \beta, \theta) ;\left(\begin{array}{c}
0 \\
-1
\end{array}\right)\right]<0, \quad \theta \in\left[-\frac{\pi}{2}, \frac{\pi}{2}\right],
$$

and so, using (49) and (50),

$$
u\left[\pi ;\left(\begin{array}{l}
0 \\
1
\end{array}\right)\right]<0 \text { and } u\left[\pi ;\left(\begin{array}{c}
0 \\
-1
\end{array}\right)\right]<0
$$

An immediate consequence of (54) is that problem (48) has no solution if $\sigma>$ 0 .

If $\sigma=\sigma_{0}<0$, there are two positive numbers $s_{1}$ and $s_{2}$ (initial slope magnitudes) uniquely defined by the relations

$$
s_{1} u\left[\pi ;\left(\begin{array}{l}
0 \\
1
\end{array}\right)\right]=\sigma_{0} \text { and } s_{2} u\left[\pi ;\left(\begin{array}{c}
0 \\
-1
\end{array}\right)\right]=\sigma_{0} .
$$

Set $w_{1}(t) \stackrel{\text { def }}{=} s_{1} u\left[t ;\left(\begin{array}{l}0 \\ 1\end{array}\right)\right]$ and $w_{2}(t) \stackrel{\text { def }}{=} s_{2} u\left[t ;\left(\begin{array}{c}0 \\ -1\end{array}\right)\right]$ for $t \in[0, \pi]$. Then $w_{1}$ and $w_{2}$ are solutions of (48) with $\sigma=\sigma_{0}<0$. Furthermore, $\dot{w}_{1}(0)=s_{1}>0$ and $\dot{w}_{2}(0)=-s_{2}<0$, so these solutions are distinct. This shows that (48) has at least two solutions whenever $\sigma<0$.

To verify that $w_{1}$ and $w_{2}$ are the only solutions of (48), let $w(t)$ be a solution; then

$$
\left(\begin{array}{c}
w(t) \\
\dot{w}(t)
\end{array}\right)=\left(\begin{array}{c}
u \\
v
\end{array}\right)\left[t ;\left(\begin{array}{c}
0 \\
\dot{w}(0)
\end{array}\right)\right], \quad t \in[0, \pi] .
$$

An argument as in the last section shows that if $\dot{w}(0)<0$ then $-\dot{w}(0)=s_{2}$ and if $\dot{w}(0)>0$ then $\dot{w}(0)=s_{1}$. Hence, either $w=w_{1}$ or $w=w_{2}$, which shows (48) to have exactly two distinct solutions for each boundary condition $\sigma<0$.

Again, from the way in which $R(I I)$ was defined, it follows that problem (48) has no solution if $\sigma>0$, only the trivial solution if $\sigma=0$, and if $\sigma<0$, precisely two solutions, characterized by the sign of their initial slopes.

7.3. Fix $(\alpha, \beta) \in R(I I I)$. From $\S 6.3$

$$
u\left[\tau_{+}\left(\alpha, \beta, \frac{\pi}{2}\right) ;\left(\begin{array}{c}
0 \\
-1
\end{array}\right)\right]<0 \text { and } u\left[\tau_{+}\left(\alpha, \beta,-\frac{\pi}{2}\right) ;\left(\begin{array}{c}
0 \\
-1
\end{array}\right)\right]>0
$$

and hence, from (49) and (50),

$$
u\left[\pi ;\left(\begin{array}{l}
0 \\
1
\end{array}\right)\right]<0 \text { and } u\left[\pi ;\left(\begin{array}{c}
0 \\
-1
\end{array}\right)\right]>0
$$


At this point the argument proceeds analogously to the argument in $\S 7.1$ and the same conclusions are obtained. That is, $(\alpha, \beta) \in R(I I I)$ implies that problem (48) has a unique solution for each $\sigma \in \mathbf{R}$.

7.4. Now we consider a boundary case. Choose $(\alpha, \beta)$ such that $\alpha<\beta$ and $k / \sqrt{\alpha}+(k+1) / \sqrt{\beta}=1$. So $(\alpha, \beta) \in \partial R(I) \cap \partial R(I I)$ and from $\S 4$.

$$
u\left[\tau_{+}\left(\alpha, \beta,-\frac{\pi}{2}\right) ;\left(\begin{array}{c}
0 \\
-1
\end{array}\right)\right]<0 \text { and } u\left[\tau_{+}\left(\alpha, \beta, \frac{\pi}{2}\right) ;\left(\begin{array}{c}
0 \\
-1
\end{array}\right)\right]=0 \text {. }
$$

From (49) and (50)

$$
u\left[\pi ;\left(\begin{array}{c}
0 \\
-1
\end{array}\right)\right]<0 \text { and } u\left[\pi ;\left(\begin{array}{l}
0 \\
1
\end{array}\right)\right]=0 .
$$

So if $\sigma>0$, problem (48) has no solution. Also, when shooting with a nonnegative initial slope one always hits $\sigma=0$. More precisely,

is a solution of (48) for each $s>0$.

$$
w_{s}(t) \stackrel{\text { def }}{=} s u\left[t ;\left(\begin{array}{l}
0 \\
1
\end{array}\right)\right]
$$

If $\sigma=\sigma_{0}<0$ then, as argued before,

$$
w(t) \stackrel{\text { def }}{=} s_{0} u\left[t ;\left(\begin{array}{c}
0 \\
-1
\end{array}\right)\right]
$$

is the unique solution of (48), where $s_{0}>0$ is defined by

$$
s_{0} u\left[\pi ;\left(\begin{array}{c}
0 \\
-1
\end{array}\right)\right]=\sigma_{0} \text {. }
$$

We conclude that problem (48) has no solution if $\sigma>0$, infinitely many solutions, all with nonnegative initial slopes, when $\sigma=0$, and a unique solution, having a negative initial slope, for each $\sigma<0$.

7.5. In the case that $(\alpha, \beta) \in \partial R(I I) \cap \partial R(I I I)$ with $\alpha<\beta$, then, for some $k=0,1,2, \ldots$,

$$
(k+1) / \sqrt{\alpha}+k / \sqrt{\beta}=1 .
$$

From $\S 6.5$

$$
u\left[\tau_{+}\left(\alpha, \beta, \frac{\pi}{2}\right) ;\left(\begin{array}{c}
0 \\
-1
\end{array}\right)\right]<0 \text { and } u\left[\tau_{+}\left(\alpha, \beta,-\frac{\pi}{2}\right) ;\left(\begin{array}{c}
0 \\
-1
\end{array}\right)\right]=0
$$

and hence

$$
u\left[\pi ;\left(\begin{array}{l}
0 \\
1
\end{array}\right)\right]<0 \text { and } u\left[\pi ;\left(\begin{array}{c}
0 \\
-1
\end{array}\right)\right]=0
$$

The argument of $\S 7.4$ shows problem (48) to have no solution if $\sigma>0$, infinitely many solutions if $\sigma=0$ (with negative initial slopes if they are nontrivial), and a unique solution, with positive initial slope, if $\sigma<0$.

\section{THE UNPERTURBED PROBLEM}

We combine and summarize these results in the theorems of this section. We assume that a nonnegative integer $k$ has been chosen and the regions $R(I)$, $R(I I)$, and $R(I I I)$ are defined as usual. 
Theorem 2. If $(\alpha, \beta) \in R(I) \cup R(I I I)$ then problem (2) has a unique solution for each pair of boundary conditions $\sigma_{1}$ and $\sigma_{2}$.

Proof. For such $\alpha$ and $\beta$ we have shown that problems (3), (4), and (5) have unique solutions for each $\sigma \in \mathbf{R}$. The theorem follows from the discussion in $\S 2$.

Theorem 3. Let $(\alpha, \beta) \in R(I I)$ with the numbers $M_{+}(\alpha, \beta)$ and $M_{-}(\alpha, \beta)$ defined as in $\S 6.2$. Define

$$
L\left(\sigma_{1}\right) \stackrel{\text { def }}{=}\left\{\begin{array}{l}
M_{+}(\alpha, \beta) \sigma_{1} \quad \text { if } \sigma_{1} \geq 0 \\
-M_{-}(\alpha, \beta) \sigma_{1} \quad \text { if } \sigma_{1}<0
\end{array}\right.
$$

and the open sets

$$
\begin{aligned}
& \mathscr{O}_{0} \stackrel{\text { def }}{=}\left\{\left(\sigma_{1}, \sigma_{2}\right) \mid \sigma_{2}>L\left(\sigma_{1}\right)\right\}, \\
& \mathscr{O}_{2} \stackrel{\text { def }}{=}\left\{\left(\sigma_{1}, \sigma_{2}\right) \mid \sigma_{2}<L\left(\sigma_{1}\right)\right\} .
\end{aligned}
$$

Then $M_{-}(\alpha, \beta)=1 / M_{+}(\alpha, \beta)$ and problem (2),

$$
\left\{\begin{array}{l}
u^{\prime \prime}+\alpha u^{-}+\beta u^{+}=0, \\
u(0)=\sigma_{1}, \quad u(\pi)=\sigma_{2},
\end{array}\right.
$$

has no solution if $\left(\sigma_{1}, \sigma_{2}\right) \in \mathscr{O}_{0}$, exactly two solutions if $\left(\sigma_{1}, \sigma_{2}\right) \in \mathscr{O}_{2}$, and a unique solution if $\sigma_{2}=L\left(\sigma_{1}\right)$.

Proof. If $\sigma_{1}>0$ and $\sigma_{2}=M_{+}(\alpha, \beta) \sigma_{1}$, the discussion in $\S 6.2$ shows that problem (3) has a unique solution for $\sigma=\sigma_{2} / \sigma_{1}$ and hence, from $\S 2$, problem (2) has a unique solution. If $\sigma_{2}=0=\sigma_{1}$, then, from the way in which $R(I I)$ is defined, problem (2) has but the trivial solution. This shows unique solvability of problem (2) whenever $\sigma_{2}=L\left(\sigma_{1}\right)$ and $\sigma_{1} \geq 0$.

Now, for each solution $u$ of problem (2) with $u(0)=\sigma_{1}$ and $u(\pi)=\sigma_{2}$ there is a corresponding solution $v$, with $v(0)=\sigma_{2}$ and $v(\pi)=\sigma_{1}$, obtained from $u$ by setting

$$
v(x)=u(\pi-x), \quad 0 \leq x \leq \pi,
$$

and, of course, the converse. Thus, by reflecting the half line $\sigma_{2}=L\left(\sigma_{1}\right)$, $\sigma_{1} \geq 0$, through the diagonal,

$$
D \stackrel{\text { def }}{=}\left\{\left(\sigma_{1}, \sigma_{2}\right) \mid \sigma_{1}=\sigma_{2}\right\},
$$

we obtain another half line $\sigma_{2}=(1 / M+1(\alpha, \beta)) \sigma_{1}, \sigma_{1} \leq 0$, containing points $\left(\sigma_{1}, \sigma_{2}\right)$ corresponding to unique solutions of (2). Moreover, from $\S 6.2$, problem (5) has unique solutions in $R(I I)$ if and only if $\sigma=M_{-}(\alpha, \beta)$ and hence problem (2) has unique solutions when $\sigma_{1}<0$ if and only if $\sigma_{2} / \sigma_{1}=-M_{-}(\alpha, \beta)$, that is, if and only if $\sigma_{2}=-M_{-}(\alpha, \beta) \sigma_{1}$. Consequently, $M_{-}(\alpha, \beta)=-1 / M_{+}(\alpha, \beta)$.

Now suppose the point $\left(\sigma_{1}, \sigma_{2}\right)$ belongs to the region $\mathscr{O}_{0}$. Then $\sigma_{1}>0$ implies that $\sigma_{2} / \sigma_{1}>M_{+}(\alpha, \beta), \sigma_{1}=0$ implies that $\sigma_{2}>0$, and $\sigma_{1}<0$ implies that $-\sigma_{2} / \sigma_{1}>M_{-}(\alpha, \beta)$. In view of $\S 6.2$, we see that problems (3) 
and (5) have no solutions and so neither does problem (2). From $\S 7.2$ problem (4), and hence problem (2), have no solution if $\sigma=\sigma_{2}>0$.

If $\left(\sigma_{1}, \sigma_{2}\right) \in \mathscr{O}_{2}$ then $\sigma_{1}>0$ implies that $\sigma_{2} / \sigma_{1}<M_{+}(\alpha, \beta), \sigma_{1}=0$ implies that $\sigma_{2}<0$, and $\sigma_{1}<0$ implies that $-\sigma_{2} / \sigma_{1}<M_{-}(\alpha, \beta)$. The discussions in $\S \S 6.2$ and 7.2 show that each of the problems (3), (4), and (5) has precisely two solutions, hence so does problem (2).

See Figure 15 for a picture of the regions $\mathscr{O}_{0}$ and $\mathscr{O}_{2}$.

The next theorem takes care of the boundary situations.

Theorem 4. Let $(\alpha, \beta) \in \partial R(I) \cap \partial R(I I)$ or $\partial R(I I) \cap \partial R(I I)$ with $\alpha<\beta$. Then problem (2) has a unique solution whenever $\sigma_{2}<-\sigma_{1}$. If $\sigma_{1} \neq 0$ and $\sigma_{2} \geq \sigma_{1}$, then (2) has no solution. Or if $\sigma_{1}=0$ and $\sigma_{2}>0$, then again (2) has no solution.

Proof. Suppose that $\sigma_{1}>0$ and put $\sigma=\sigma_{2} / \sigma_{1}$; then from $\S \S 6.4$ and 6.5 we see that problem (3) has a unique solution if $\sigma<-1$ and no solution if $\sigma \geq-1$. Hence, problem (2) in this case has a unique solution if $\sigma_{2}<-\sigma_{1}$ and no solution if $\sigma_{2} \geq-\sigma_{1}$. If $\sigma_{1}<0$, we can argue the same way.

If $\sigma_{1}=0$, problem (4), and hence problem (2), has a unique solution if $\sigma=\sigma_{2}<0$ and no solution if $\sigma=\sigma_{2}>0$.

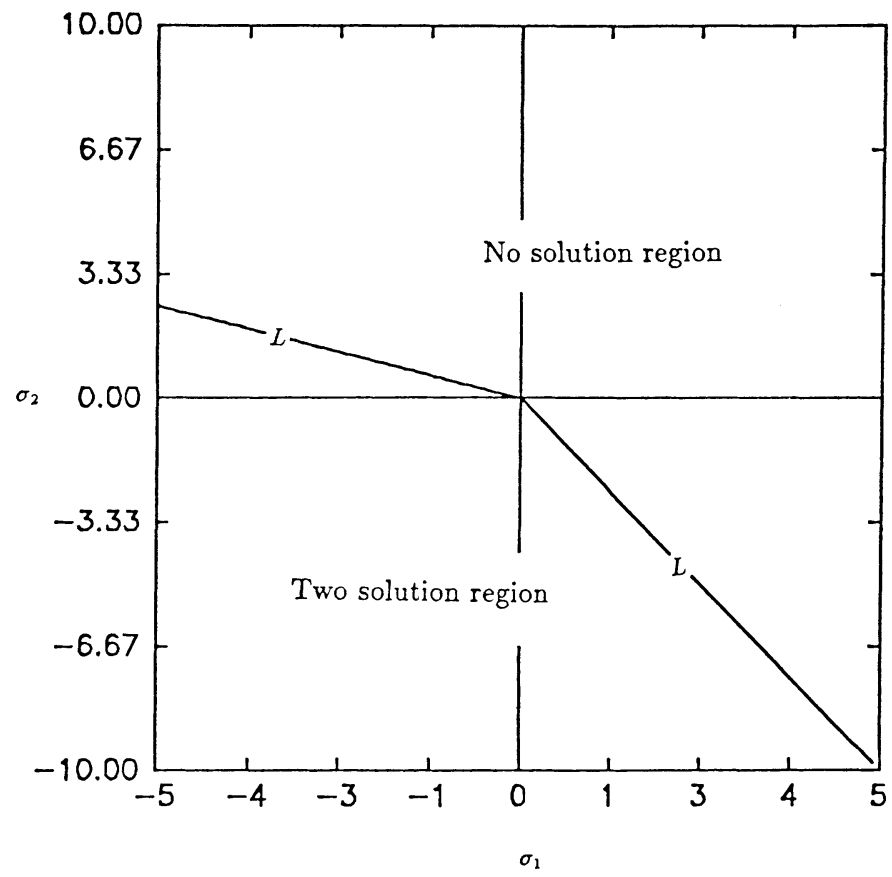

FIGURE 15. Solvability regions in the $\sigma_{1} \sigma_{2}$-plane for problem (2) when $(\alpha, \beta) \in R(I I)$. If the pair $\left(\sigma_{1}, \sigma_{2}\right)$ lies on the broken line $L$, problem (2) has a unique solution. 


\section{Convergence AND Blowup}

We are ready to put together a few results that will be useful in computing the local degrees (indices) of the solutions obtained for the unperturbed problems (3), (4), and (5). It is again assumed that $k \in\{0,1,2, \ldots\}$ is fixed, thus the regions $R(I), R(I I)$, and $R(I I I)$ defined, and $\left(\alpha_{0}, \beta_{0}\right) \in \partial R(I) \cap \partial R(I I)$ with $\alpha_{0}<\beta_{0}$.

Recall that, if $(\alpha, \beta) \in R(I I)$, the functions $\sigma_{+}(\alpha, \beta, \cdot)$ and $\sigma_{-}(\alpha, \beta, \cdot)$ have unique critical points, which we will call $\theta_{+}(\alpha, \beta)$ and $\theta_{-}(\alpha, \beta)$, respectively, in the open interval $(-\pi / 2, \pi / 2)$.

Lemma 5. $\theta_{ \pm}(\alpha, \beta) \rightarrow \pi / 2$ as the point $(\alpha, \beta)$ approaches $\left(\alpha_{0}, \beta_{0}\right)$ through values in $R(I I)$.

Proof. Recall, for $(\alpha, \beta) \in R(I I)$, that $\theta_{+}(\alpha, \beta)$ and $\theta_{-}(\alpha, \beta)$ are the unique zeros in $[-\pi / 2, \pi / 2]$ of $g_{+}(\alpha, \beta, \theta)$ and $g_{-}(\alpha, \beta, \theta)$, respectively. Now suppose there is a sequence $\left\{\left(\alpha_{j}, \beta_{j}\right)\right\}_{j=1}^{\infty} \subset R(I I)$ and an $\varepsilon>0$ such that

$$
\left(\alpha_{j}, \beta_{j}\right) \stackrel{R(I I)}{\longrightarrow}\left(\alpha_{0}, \beta_{0}\right)
$$

but

$$
-\pi / 2<\theta_{ \pm}\left(\alpha_{j}, \beta_{j}\right) \leq \pi / 2-\varepsilon \text { for each } j=1,2,3, \ldots
$$

Let $\bar{\theta}_{-}$and $\bar{\theta}_{+}$denote limit points of the sequences $\left\{\theta_{-}\left(\alpha_{j}, \beta_{j}\right)\right\}$ and $\left\{\theta_{+}\left(\alpha_{j}, \beta_{j}\right)\right\}$, respectively. Then $\bar{\theta}_{ \pm} \in[-\pi / 2, \pi / 2-\varepsilon]$ and from continuity of

we find that

$$
g_{ \pm}: Q^{+} \times[-\pi / 2, \pi / 2] \rightarrow \mathbf{R}
$$

$$
0=g_{ \pm}\left(\alpha_{0}, \beta_{0}, \bar{\theta}_{ \pm}\right)
$$

However, $g_{ \pm}\left(\alpha_{0}, \beta_{0}, \cdot\right)$ has $\theta=\pi / 2$ as its unique zero in $[-\pi / 2, \pi / 2]$ (cf. $6.4)$, which contradicts (55) and so the lemma is proved.

For each pair $(\alpha, \beta) \in Q^{+}$the notation

$$
u_{+}(\cdot ; \alpha, \beta, \theta), \quad \theta \in(-\pi / 2, \pi / 2),
$$

is used to denote the solution of problem (3) with $\sigma=\sigma_{+}(\alpha, \beta, \theta)$. Hence,

$$
u_{+}(\cdot ; \alpha, \beta, \theta)=u\left[\cdot ;\left(\begin{array}{c}
\frac{1}{\sqrt{\beta}} \cos \theta \\
\sin \theta
\end{array}\right)\right] / \frac{1}{\sqrt{\beta}} \cos \theta \text {. }
$$

For $r>0$ set

$$
B_{r}\left(\alpha_{0}, \beta_{0}\right) \stackrel{\text { def }}{=}\left\{(\alpha, \beta) \mid\left(\alpha-\alpha_{0}\right)^{2}+\left(\beta-\beta_{0}\right)^{2}<r^{2}\right\}
$$

and define the point $q=q(r)$ by

$$
q(r) \stackrel{\text { def }}{=} \inf \left\{\theta_{+}(\alpha, \beta) \mid(\alpha, \beta) \in B_{r}\left(\alpha_{0}, \beta_{0}\right) \cap R(I I)\right\} .
$$

From Lemma 5 we see that $q(r) \rightarrow \pi / 2$ as $r \rightarrow 0$. Fix $\theta_{0} \in(-\pi / 2, \pi / 2)$ and pick $r_{0}>0$ and sufficiently small that $\theta_{0}<q\left(r_{0}\right)$. 
Proposition 6. Suppose that $(\alpha, \beta) \rightarrow\left(\alpha_{0}, \beta_{0}\right)$; then $u_{+}\left(\cdot ; \alpha, \beta, \theta_{0}\right)$ converges to $u_{+}\left(\cdot ; \alpha_{0}, \beta_{0}, \theta_{0}\right)$ in $C^{1}[0, \pi]$.

Proof. If not, then there is a sequence $\left\{\left(\alpha_{j}, \beta_{j}\right)\right\}_{j=1}^{\infty} \subset B_{r_{0}}\left(\alpha_{0}, \beta_{0}\right)$ and $\varepsilon>0$ such that

and

$$
\left(\alpha_{j}, \beta_{j}\right) \rightarrow\left(\alpha_{0}, \beta_{0}\right)
$$

$$
\left\|u_{+}\left(\cdot ; \alpha_{j}, \beta_{j}, \theta_{0}\right)-u_{+}\left(\cdot ; \alpha_{0}, \beta_{0}, \theta_{0}\right)\right\|_{1} \geq \varepsilon, \quad j=1,2,3, \ldots,
$$

with $r_{0}>0$ is defined as above and $\|\cdot\|_{1}$ the norm on $C^{1}[0, \pi]$.

For notational convenience define the maps

$$
\begin{aligned}
& l_{j}(x) \stackrel{\text { def }}{=}((\pi-x) / \pi)+\sigma_{+}\left(\alpha_{j}, \beta_{j}, \theta_{0}\right)(x / \pi), \quad 0 \leq x \leq \pi, \\
& f_{j}(u) \stackrel{\text { def }}{=} \alpha_{j} u^{-}+\beta_{j} u^{+}, \\
& u_{j}(\cdot) \stackrel{\text { def }}{=} u_{+}\left(\cdot ; \alpha_{j}, \beta_{j}, \theta_{0}\right), \quad j=1,2,3, \ldots
\end{aligned}
$$

Then for each $j$

$$
u_{j}=K f_{j}\left(u_{j}\right)+l_{j},
$$

where $K$ is the solution operator (i.e., the Green's operator) associated with the linear boundary value problem

$$
-u^{\prime \prime}(x)=h(x), \quad 0 \leq x \leq \pi, u(0)=u(\pi)=0 .
$$

From (56) we have, for some $M>0$, the bound

$$
\left\|u_{j}\right\|_{1} \leq M, \quad j=1,2,3, \ldots
$$

Consequently,

$$
\left\|f_{j}\left(u_{j}\right)\right\|_{0} \leq\left(\max _{j} \alpha_{j}+\max _{j} \beta_{j}\right)\left\|u_{j}\right\|_{1} \leq \widetilde{M}
$$

for some $\widetilde{M}>0$, where $\|\cdot\|_{0}$ is used to denote the norm on $C^{0}[0, \pi]=$ $C[0, \pi]$. Using $(58)$ and the compactness of the operator $K: C^{0}[0, \pi] \rightarrow$ $C^{1}[0, \pi]$, we may assume that

$$
u_{j} \stackrel{C^{1}}{\rightarrow} v \quad \text { as } j \rightarrow \infty
$$

for some $v \in C^{1}[0, \pi]$. Thus,

$$
f_{j}\left(u_{j}\right)=\alpha_{j} u_{j}^{-}+\beta_{j} u_{j}^{+} \stackrel{C^{0}}{\rightarrow} \alpha_{0} v^{-}+\beta_{0} v^{+}
$$

as $j \rightarrow \infty$, and again from (58) we see that $v$ satisfies

$$
v=K\left(\alpha_{0} v^{-}+\beta_{0} v^{+}\right)+l_{0} .
$$

Equivalently, $v \in C^{2}[0, \pi]$ and

$$
\left\{\begin{array}{l}
v^{\prime \prime}+\alpha_{0} v^{-}+\beta_{0} v^{+}=0 \\
v(0)=1, \quad v(\pi)=\sigma_{+}\left(\alpha_{0}, \beta_{0}, \theta_{0}\right) .
\end{array}\right.
$$


So $v$ is a solution of problem (3) with $(\alpha, \beta)=\left(\alpha_{0}, \beta_{0}\right)$ and $\sigma=\sigma_{+}\left(\alpha_{0}, \beta_{0}, \theta_{0}\right)$. But this problem has the unique solution $u_{+}\left(\cdot ; \alpha_{0}, \beta_{0}, \theta_{0}\right)$.

If the notation

$$
u_{-}(\cdot ; \alpha, \beta, \theta), \quad \theta \in(-\pi / 2, \pi / 2),
$$

is used to denote the solution of problem (5) with $\sigma=\sigma_{-}(\alpha, \beta, \theta)$, it follows that

$$
u_{-}(\cdot ; \alpha, \beta, \theta)=u\left[\cdot ;\left(\begin{array}{c}
-\frac{1}{\sqrt{\alpha}} \cos \theta \\
\sin \theta
\end{array}\right)\right] / \frac{1}{\sqrt{\alpha}} \cos \theta
$$

and an argument analogous to the one used in Proposition 6 gives us

Proposition 7. If $(\alpha, \beta) \rightarrow\left(\alpha_{0}, \beta_{0}\right)$, then

$$
u_{-}\left(\cdot ; \alpha, \beta, \theta_{0}\right) \stackrel{C^{1}}{\longrightarrow} u_{-}\left(\cdot ; \alpha_{0}, \beta_{0}, \theta_{0}\right) .
$$

Remark 1. Let $(\alpha, \beta) \in R(I)$. If $\sigma_{0} \geq-1$ and $\psi_{+}(\alpha, \beta) \in(-\pi / 2, \pi / 2)$ denotes the unique point such that $\sigma_{+}\left(\alpha, \beta, \psi_{+}(\alpha, \beta)\right)=\sigma_{0}$, then

$$
\left\|u_{+}\left(\cdot ; \alpha, \beta, \psi_{+}(\alpha, \beta)\right)\right\|_{1} \rightarrow \infty
$$

as $(\alpha, \beta) \rightarrow\left(\alpha_{0}, \beta_{0}\right)$. Similarly, if $\sigma_{0} \geq 1$ and $\psi_{-}(\alpha, \beta) \in(-\pi / 2, \pi / 2)$ denotes the unique point such that $\sigma_{-}\left(\alpha, \beta, \psi_{+}(\alpha, \beta)\right)=\sigma_{0}$, then

$$
\left\|u_{-}\left(\cdot ; \alpha, \beta, \psi_{-}(\alpha, \beta)\right)\right\|_{1} \rightarrow \infty
$$

as $(\alpha, \beta) \rightarrow\left(\alpha_{0}, \beta_{0}\right)$.

To see this, we argue the case for $u_{+}$(the case for $u_{-}$is similar). If the solutions

$$
u_{+}\left(\cdot ; \alpha, \beta, \psi_{+}(\alpha, \beta)\right)
$$

(or some subsequence of them) were bounded in $C^{1}[0, \pi]$, we would have, as in the proof of Proposition 6, the existence of some $v \in C^{2}[0, \pi]$ satisfying

$$
\left\{\begin{array}{l}
v^{\prime \prime}+\alpha_{0} v^{-}+\beta_{0} v^{+}=0 \\
v(0)=1, \quad v(\pi)=\sigma_{0} .
\end{array}\right.
$$

But problem (3) with $\sigma=\sigma_{0} \geq-1$ has no solution.

Remark 2. For $(\alpha, \beta) \in R(I I)$ and close to $\left(\alpha_{0}, \beta_{0}\right)$ consider the set

$$
\mathscr{S}_{+}(\alpha, \beta) \stackrel{\text { def }}{=}\left\{u_{+}(\cdot ; \alpha, \beta, \theta) \mid \theta_{+}(\alpha, \beta)<\theta<\pi / 2\right\} .
$$

From (56) we have

and hence the estimate

$$
\dot{u}_{+}(0 ; \alpha, \beta, \theta)=\sqrt{\beta} \tan \theta
$$

$$
\left\|u_{+}(\cdot ; \alpha, \beta, \theta)\right\|_{1}>\sqrt{\beta} \tan \theta_{+}(\alpha, \beta)
$$

holds whenever $u_{+}(\cdot, \alpha, \beta, \theta) \in \mathscr{S}_{+}(\alpha, \beta)$ and $(\alpha, \beta)$ is sufficiently close to $\left(\alpha_{0}, \beta_{0}\right)$. Since

$$
\theta_{+}(\alpha, \beta) \rightarrow \pi / 2 \text { as }(\alpha, \beta) \stackrel{R(I I)}{\longrightarrow}\left(\alpha_{0}, \beta_{0}\right)
$$


it follows that each solution in $\mathscr{S}_{+}(\alpha, \beta)$ becomes unbounded as $(\alpha, \beta) \stackrel{R(I I)}{\longrightarrow}$ $\left(\alpha_{0}, \beta_{0}\right)$. Of course, we have the analogous result for $u_{-}$, i.e.,

$$
\left\|u_{-}(\cdot ; \alpha, \beta, \theta)\right\|_{1} \rightarrow \infty, \quad \text { as }(\alpha, \beta) \stackrel{R(I I)}{\longrightarrow}\left(\alpha_{0}, \beta_{0}\right)
$$

provided $0_{-}(\alpha, \beta)<\theta<\pi / 2$.

Remark 3. If we think of the graphs of $\sigma_{ \pm}(\alpha, \beta, \cdot)$, for $(\alpha, \beta) \in R(I I)$, as defining two branches of solutions, a left branch and a right branch, for each of problems (3) and (5), then we may combine the above two remarks into a single observation. That is, solutions corresponding to the right branch become unbounded as $(\alpha, \beta)$ approaches $\left(\alpha_{0}, \beta_{0}\right)$ from $R(I I)$. Moreover, in view of Proposition 6, the graphs (unique branches) defined by $\sigma_{ \pm}\left(\alpha_{0}, \beta_{0}, \cdot\right)$ are the limits, in some sense, of the graphs of $\sigma_{ \pm}(\alpha, \beta, \cdot)$ as $(\alpha, \beta)$ approaches $\left(\alpha_{0}, \beta_{0}\right)$. One sees that the right branches of $\sigma_{ \pm}(\alpha, \beta, \cdot),(\alpha, \beta) \in R(I I)$, and the "upper" parts of the graphs of $\sigma_{ \pm}(\alpha, \beta, \cdot),(\alpha, \beta) \in R(I)$, "disappear," i.e., the associated solutions become unbounded in the sense of the above two remarks, as we pass to this limit.

\section{THE INDEX OF THE TRIVIAL SOlUtion}

Consider again the homogeneous problem

$$
\left\{\begin{array}{l}
u^{\prime \prime}+\alpha u^{-}+\beta u^{+}=0 \\
u(0)=u(\pi)=0 .
\end{array}\right.
$$

Let $A \subset Q^{+}$be defined by

$$
A \stackrel{\text { def }}{=}\left\{(\alpha, \beta) \in Q^{+} \mid \text {problem (5) has a nontrivial solution }\right\} \text {. }
$$

Evidently then, $(\alpha, \beta) \in A$ if and only if $(\alpha, \beta)$ is on one of the curves (22), (23), or (24).

If $(\alpha, \beta) \in Q^{+} \backslash A$, then (59) has only the trivial solution and so the trivial function is the only solution of

$$
u=K\left[\alpha u^{-}+\beta u^{+}\right], \quad u \in C^{1}[0, \pi] .
$$

Thus, if $B_{r} \subset C^{1}[0, \pi]$ denotes the ball of radius $r>0$ centered at $u \equiv 0$, then

$$
\operatorname{deg}_{L S}\left(\mathrm{id}-K\left[\alpha(\cdot)^{-}+\beta(\cdot)^{+}\right], B_{r}, 0\right)
$$

is defined and independent of $r>0$. In particular, if $(\alpha, \beta) \in Q^{+} \backslash A$, then the index of the trivial solution, denoted by $\operatorname{ind}(0 ; \alpha, \beta)$, is defined by

$$
\operatorname{ind}(0 ; \alpha, \beta) \stackrel{\text { def }}{=} \operatorname{deg}_{L S}\left(\text { id }-K\left[\alpha(\cdot)^{-}+\beta(\cdot)^{+}\right], B_{r}, 0\right) \text {. }
$$

So we have a map

$$
\operatorname{ind}(0 ; \cdot, \cdot): Q^{+} / A \rightarrow \mathbf{Z},
$$

where the symbol $\mathbf{Z}$ is used to represent the integers. Moreover, since

$$
\left\|\left(\alpha u^{-}+\beta u^{+}\right)-\left(\bar{\alpha} u^{-}+\bar{\beta} u^{+}\right)\right\|_{0} \leq(|\alpha-\bar{\alpha}|+|\beta-\bar{\beta}|)\|u\|_{0},
$$


for $u \in \bar{B}_{r}$

$$
\begin{aligned}
\left\|\left(u-K\left[\alpha u^{-}+\beta u^{+}\right]\right)-\left(u-K\left[\bar{\alpha} u^{-}+\bar{\beta} u^{+}\right]\right)\right\|_{1} \\
\quad \leq\|K\|_{0,1}\left\|\left(\alpha u^{-}+\beta u^{+}\right)-\left(\bar{\alpha} u^{-}+\bar{\beta} u^{+}\right)\right\|_{0} \\
\quad \leq\|K\|_{0,1}(|\alpha-\bar{\alpha}|+|\beta-\bar{\beta}|)\|u\|_{0} \\
\quad \leq\|K\|_{0,1}(|\alpha-\bar{\alpha}|+|\beta-\bar{\beta}|)\|u\|_{1} \\
\quad \leq(|\alpha-\bar{\alpha}|+|\beta-\bar{\beta}|)\|K\|_{0,1} r
\end{aligned}
$$

where $\|\cdot\|_{0,1}$ is used to denote the norm on the Banach space of bounded linear transformations from $C^{0}[0, \pi]$ into $C^{1}[0, \pi]$. The map

$$
u \mapsto u-K\left[\alpha u^{-}+\beta u^{+}\right]
$$

is then subject to uniform approximation on $\bar{B}_{r}$ by maps of the form

$$
\mathrm{id}-K\left[\bar{\alpha}(\cdot)^{-}+\bar{\beta}(\cdot)^{+}\right]: C^{1}[0, \pi] \rightarrow C^{1}[0, \pi]
$$

provided that $(\bar{\alpha}, \bar{\beta})$ is close to $(\alpha, \beta)$. This shows that, for fixed $r>0$,

$$
\operatorname{deg}_{L S}\left(\mathrm{id}-K\left[\alpha(\cdot)^{-}+\beta(\cdot)^{+}\right], B_{r}, 0\right)=\operatorname{deg}_{L S}\left(\mathrm{id}-K\left[\bar{\alpha}(\cdot)^{-}+\bar{\beta}(\cdot)^{+}\right], B_{r}, 0\right)
$$

and hence

$$
\operatorname{ind}(0 ; \alpha, \beta)=\operatorname{ind}(0 ; \bar{\alpha}, \bar{\beta})
$$

whenever $(\bar{\alpha}, \bar{\beta})$ is sufficiently close to $(\alpha, \beta)$. Consequently, the index of the trivial solution is a continuous map

$$
\operatorname{ind}(0 ; \cdot, \cdot): Q^{+} \backslash A \rightarrow \mathbf{Z}
$$

and hence is constant on the connected components of $Q^{+} \backslash A$. In particular, $\operatorname{ind}(0 ; \cdot, \cdot)$ is constant on each of the regions $R(I), R(I I)$, and $R(I I I)$.

If $(\alpha, \beta)$ belongs to $R(I)$, then to compute the value of this index we first note that the diagonal $\left\{(\alpha, \beta) \in Q^{+} \mid \alpha=\beta\right\}$ has nonempty intersection with the connected component of $Q^{+} / A$ containing $R(I)$ and so

$$
\operatorname{ind}(0 ; \alpha, \beta)=\operatorname{ind}(0 ; \lambda, \lambda)=\operatorname{deg}_{L S}\left(\mathrm{id}-\lambda K, B_{r}, 0\right)=(-1)^{2 k}=1
$$

since $(2 k)^{2}<\lambda<(2 k+1)^{2}$. Similarly, if $(\alpha, \beta) \in R(I I I)$.

$$
\text { ind }(0 ; \alpha, \beta)=(-1)^{2 k+i}=-1 \text {. }
$$

The following technical lemma will allow us to obtain solutions of the problem

$$
\left\{\begin{array}{l}
u^{\prime \prime}(x)+f(u(x))=h(x), \\
u(0)=\sigma_{1}, \quad u(\pi)=\sigma_{2} .
\end{array}\right.
$$

Lemma 8. Fix $r>0$ and $(\alpha, \beta) \in Q^{+}$. Define

$$
f_{t}(u)=\alpha u^{-}+\beta u^{+} \nu(t u) / t
$$


for each $u \in C^{1}[0, \pi]$ and $t>0$. Then

$$
\left\|K f_{t}(u)-K\left[\alpha u^{-}+\beta u^{+}\right]\right\|_{1} \rightarrow 0
$$

uniformly on $\bar{B}_{r}$ as $t \rightarrow \infty$.

Proof. First we have the estimate

$$
\left\|K f_{t}(u)-K\left[\alpha u^{-}+\beta u^{+}\right]\right\|_{1}=\|K \nu(t u) / t\|_{1} \leq\|K\|_{0,1}\|\nu(t u) / t\|_{0} .
$$

Since the perturbation $\nu$ is $o(s)$ as $|s| \rightarrow \infty$ (cf. $\S 1$ ), one may argue that

$$
\|\nu(t u) / t\|_{0} \rightarrow 0
$$

uniformly on $\bar{B}_{r}$ as $t \rightarrow \infty$. The lemma follows from the estimate (61).

Theorem 9. Let $(\alpha, \beta) \in R(I) \cup R(I I I)$; then for each $h \in C[0, \pi]$ and $\sigma_{1}$, $\sigma_{2}$ in $\mathbf{R}$ the problem $(60)$

has a solution.

$$
\left\{\begin{array}{l}
u^{\prime \prime}+f(u)=h, \\
u(0)=\sigma_{1}, \quad u(\pi)=\sigma_{2},
\end{array}\right.
$$

Proof. Set

$$
l(x)=\sigma_{1}((\pi-x) / \pi)+\sigma_{2}(x / \pi), \quad 0 \leq x \leq \pi ;
$$

then solutions of (60) may be found as solutions of the equation

$$
u=K[f(u)-h]+l, \quad u \in C^{1}[0, \pi] .
$$

Let $(\alpha, \beta) \in R(I) \cup R(I I I)$ and $r>0$ be fixed. Then

$$
\begin{array}{r}
\left\|\left\{u-K f_{t}(u)+(1 / t)(K h-l)\right\}-\left\{u-K\left[\alpha u^{-}+\beta u^{+}\right]\right\}\right\|_{1} \\
\leq\left\|K f_{t}(u)-K\left[\alpha u^{-}+\beta u^{+}\right]\right\|_{1}+(1 / t)\|K h-l\|_{1}
\end{array}
$$

and using Lemma 8 we see that the right-hand side of the above estimate tends to zero, uniformly for $u \in \bar{B}_{r}$, as $t \rightarrow \infty$. Thus, there exists a $T_{0}>0$ such that $t \geq T_{0}$ implies

$$
\begin{aligned}
\operatorname{deg}_{L S}(\mathrm{id}- & \left.K f_{t}(\cdot)+(1 / t)(K h-l), B_{r}, 0\right) \\
& =\operatorname{deg}_{L S}\left(\mathrm{id}-K\left[\alpha(\cdot)^{-}+\beta(\cdot)^{+}\right], B_{r}, 0\right)=\operatorname{ind}(0 ; \alpha, \beta) .
\end{aligned}
$$

If $(\alpha, \beta) \in R(I)$ we have already seen that this index is 1 , and if $(\alpha, \beta) \in$ $R(I I I)$ this index is -1 . In either case the index is nonzero and so, for each $t \geq T_{0}$, there is a $w_{t} \in B_{r}$ such that

$$
w_{t}=K\left(f_{t}\left(w_{t}\right)-(1 / t) h\right)+(1 / t) l .
$$

By the smoothing properties of $K, w_{t} \in C^{2}[0, \pi]$ and satisfies

$$
\left\{\begin{array}{l}
w_{t}^{\prime \prime}+\alpha w_{t}^{-}+\beta w_{t}^{+}+\nu\left(t w_{t}\right) / t=(1 / t) h \\
w_{t}(0)=\sigma_{1} / t, \quad w_{t}(\pi)=\sigma_{2} / t
\end{array}\right.
$$

It follows then that $u \stackrel{\text { def }}{=} t w_{t}$ solves problem $(60)$. 
If $(\alpha, \beta) \leq R(I)$, then to compute the index, ind $\left(u_{+}(\cdot ; \alpha, \beta, \theta)\right)$, set

$$
l(x)=((\pi-x) / \pi)+\sigma_{+}(\alpha, \beta, \theta)(x / \pi)
$$

and note that for each $t \in[0,1]$ the unique solution of the problem

$$
\left\{\begin{array}{l}
u^{\prime \prime}+\alpha u^{-}+\beta u^{+}=0, \\
u(0)=\sigma_{1}, \quad u(\pi)=\sigma_{2},
\end{array}\right.
$$

with $\sigma_{1}=t$ and $\sigma_{2}=t \sigma_{+}(\alpha, \beta, \theta)$ is just $t u_{+}(\cdot ; \alpha, \beta, \theta)$. Hence, choosing

$$
R>\| u_{+}\left(\cdot ; \alpha, \beta, \theta \|_{1}\right.
$$

we have, using $t$ as a homotopy parameter,

$$
\operatorname{deg}_{L S}\left(\mathrm{id}-K\left[\alpha(\cdot)^{-}+\beta(\cdot)^{+}\right], B_{R}, t l\right)=\operatorname{deg}_{L S}\left(\mathrm{id}-K\left[\alpha(\cdot)^{-}+\beta(\cdot)^{+}\right], B_{R}, 0\right)
$$

for all $t \in[0,1]$. Setting $t=1$ yields

$$
\operatorname{ind}\left(u_{+}(\cdot ; \alpha, \beta, \theta)\right)=\operatorname{ind}(0 ; \alpha, \beta)=1 \text {. }
$$

By similar means, if $(\alpha, \beta) \in R(I I I)$ then

$$
\operatorname{ind}\left(u_{+}(\cdot ; \alpha, \beta, \theta)\right)=\operatorname{ind}(0 ; \alpha, \beta)=-1 \text {. }
$$

As a final remark of this section we make the observation that if $(\alpha, \beta) \in$ $R(I I)$ then ind $(0 ; \alpha, \beta)=0$. This follows by noting that if $\operatorname{ind}(0 ; \alpha, \beta) \neq 0$ for some $(\alpha, \beta) \in R(I I)$ then, following the proof of Theorem 9 with $\nu \equiv 0$ and $h \equiv 0$, we would have solutions of problem (63) for each pair of boundary conditions $\sigma_{1}$ and $\sigma_{2}$. But this contradicts Theorem 3 .

\section{LOWER BOUNDS IN $\overline{R(I I)}$}

In this section we obtain some lower bounds on the number of solutions to problem $(60)$ when $(\alpha, \beta) \in \overline{R(I I)}$.

Let $(\alpha, \beta) \in R(I I)$ be fixed and, again, $\theta_{+}(\alpha, \beta) \in(-\pi / 2, \pi / 2)$ is to denote the unique critical point of $\sigma_{+}(\alpha, \beta, \cdot):(-\pi / 2, \pi / 2) \rightarrow \mathbf{R}$. Recall that

$$
M_{+}(\alpha, \beta)=\sigma_{+}\left(\alpha, \beta, \theta_{+}(\alpha, \beta)\right)=\max _{-\pi / 2<\theta<\pi / 2} \sigma_{+}(\alpha, \beta, \theta) .
$$

As we have seen, if $\sigma_{0}<M_{+}(\alpha, \beta)$, there exist two points $\zeta_{1}$ and $\zeta_{2}$ with

$$
-\pi / 2<\zeta_{1}<\theta_{+}(\alpha, \beta)<\zeta_{2}<\pi / 2
$$

and

$$
\sigma_{+}\left(\alpha, \beta, \zeta_{1}\right)=\sigma_{+}\left(\alpha, \beta, \zeta_{2}\right)=\sigma_{0} .
$$

This gave us the two solutions $u_{+}\left(\cdot ; \alpha, \beta, \zeta_{1}\right)$ and $u_{+}\left(\cdot ; \alpha, \beta, \zeta_{2}\right)$ of problem (3), defined by (cf. (56))

$$
u_{+}\left(\cdot ; \alpha, \beta, \zeta_{i}\right)=u\left[\cdot ;\left(\begin{array}{c}
\frac{1}{\sqrt{\beta}} \cos \zeta_{i} \\
\sin \zeta_{i}
\end{array}\right)\right] / \frac{1}{\sqrt{\beta}} \cos \zeta_{i}, \quad i=1,2 .
$$

Thus, $u_{+}\left(\cdot, \alpha, \beta, \zeta_{i}\right), i=1,2$, are the only solutions of

$$
u=k\left[\alpha u^{-}+\beta u^{+}\right]+l, \quad u \in C^{1}[0, \pi],
$$


where as before

$$
l(x)=((\pi-x) / \pi)+\sigma_{0}(x / \pi) .
$$

Since these solutions are isolated, each has an index. To compute these indices we will use the next lemma.

Lemma 10. Fix $(\alpha, \beta) \in R(I I)$. Then ind $\left(u_{+}(\cdot ; \alpha, \beta, \theta)\right)$ is constant for $\theta$ in the interval $-\pi / 2<\theta<\theta_{+}(\alpha, \beta)$.

Proof. Choose $\theta_{0}<\theta_{1}$ so that $\left[\theta_{0}, \theta_{1}\right] \subset\left(-\pi / 2, \theta_{+}(\alpha, \beta)\right)$. If $\theta \in\left[\theta_{0}, \theta_{1}\right]$ then from (56) we see that

$$
\dot{u}_{+}(0 ; \alpha, \beta, \theta)=\sqrt{\beta} \tan \theta
$$

and thus the norms

$$
\left\|u_{+}(\cdot ; \alpha, \beta, \theta)\right\|_{1}
$$

are bounded for $\theta \in\left[\theta_{0}, \theta_{1}\right]$. Choose

$$
R>\max _{\theta \in\left[\theta_{0}, \theta_{1}\right]}\left\|u_{+}(\cdot ; \alpha, \beta, \theta)\right\|_{1}
$$

and $\varepsilon>0$ sufficiently small to ensure that $\theta_{1}+\varepsilon<\theta_{+}(\alpha, \beta)$.

Define the open set

$$
\mathscr{U} \stackrel{\text { def }}{=}\left\{u \in C^{1}[0, \pi] \mid u^{\prime}(0)<\sqrt{\beta} \tan \left(\theta_{1}+\varepsilon\right)\right\},
$$

and set $\mathscr{N}=\mathscr{U} \cap B_{R}$, where $B_{R} \subset C^{1}[0, \pi]$ is a ball of radius $R$ centered at the trivial function. Then $\mathscr{N}$ is a bounded open set in $C^{1}[0, \pi]$. Furthermore, $u_{+}(\cdot ; \alpha, \beta, \theta) \in \mathcal{N}$ if $\theta_{0} \leq \theta \leq \theta_{1}$, and if $\theta>\theta_{+}(\alpha, \beta)$ then

$$
\dot{u}_{+}(0 ; \alpha, \beta, \theta)=\sqrt{\beta} \tan \theta>\sqrt{\beta} \tan \left(\theta_{1}+\varepsilon\right)
$$

and therefore $u_{+}(\cdot, \alpha, \beta, \theta) \notin \overline{\mathcal{N}}$. Thus, if

$$
\sigma \in\left[\sigma_{+}\left(\alpha, \beta, \theta_{0}\right), \sigma_{+}\left(\alpha, \beta, \theta_{1}\right)\right],
$$

problem (3) has only the solution

$$
u_{+}(\cdot ; \alpha, \beta, \theta) \in \overline{\mathscr{N}}
$$

for some unique $\theta$ where $\theta_{0} \leq \theta \leq \theta_{1}$ and $\sigma_{+}(\alpha, \beta, \theta)=\sigma$. If we define

$$
l_{\theta}(x) \stackrel{\text { def }}{=}\left(\frac{\pi-x}{\pi}\right)+\sigma_{+}(\alpha, \beta, \theta)\left(\frac{x}{\pi}\right), \quad \theta \in\left(-\frac{\pi}{2}, \frac{\pi}{2}\right), 0 \leq x \leq \pi,
$$

then, letting $\theta \in\left[\theta_{0}, \theta_{1}\right]$ be a homotopy parameter, we see that

$$
\operatorname{deg}_{L S}\left(\mathrm{id}-K\left[\alpha(\cdot)^{-}+\beta(\cdot)^{+}\right], \mathcal{N}, l_{\theta}\right)
$$

is defined and constant for $\theta \in\left[\theta_{0}, \theta_{1}\right]$. Since the only requirements made on $\theta_{0}$ and $\theta_{1}$ were that

$$
-\pi / 2<\theta_{0}<\theta_{1}<\theta_{+}(\alpha, \beta),
$$

the lemma is proved.

Lemma 10 shows that, given $(\alpha, \beta) \in R(I I)$, each solution associated with the left branch of $\sigma_{+}(\alpha, \beta, \cdot)$ has the same index. We will prove below that solutions associated with left branches of any of the functions $\sigma_{+}(\alpha, \beta, \cdot)$, $(\alpha, \beta) \in R(I I)$, have the same index. 
Lemma 11. Suppose that $\left(\alpha_{1}, \beta_{1}\right)$ and $\left(\alpha_{2}, \beta_{2}\right)$ are two points from region $R(I I)$. If $\theta_{1}$ and $\theta_{2}$ are such that

$$
-\pi / 2<\theta_{1}<\theta_{+}\left(\alpha_{1}, \beta_{1}\right) \text { and }-\pi / 2<\theta_{2}<\theta_{+}\left(\alpha_{2}, \beta_{2}\right),
$$

then

$$
\operatorname{ind}\left(u_{+}\left(\cdot ; \alpha_{1}, \beta_{1}, \theta_{1}\right)\right)=\operatorname{ind}\left(u_{+}\left(\cdot ; \alpha_{2}, \beta_{2}, \theta_{2}\right)\right) .
$$

Proof. First we show that the uniquely defined critical point $\theta_{+}(\alpha, \beta)$ depends continuously on $(\alpha, \beta) \in R(I I)$. We will rely on the already established fact that $\theta_{+} \in(-\pi / 2, \pi / 2)$ is the unique zero of the continuous map.

$$
g_{+}: R(I I) \times[-\pi / 2, \pi / 2] \rightarrow \mathbf{R} .
$$

Suppose that this dependence of $\theta_{+}$on $(\alpha, \beta)$ is not continuous at some point $(\alpha, \beta) \in R(I I)$. Then there are a positive $\delta$ and a sequence $\left\{\left(\alpha_{j}, \beta_{j}\right)\right\}_{j \geq 1} \in$ $R(I I)$ such that

$$
\left(\alpha_{j}, \beta_{j}\right) \stackrel{R(I I)}{\longrightarrow}(\alpha, \beta)
$$

but

$$
\left|\theta_{+}\left(\alpha_{j}, \beta_{j}\right)-\theta_{+}(\alpha, \beta)\right| \geq \delta \text { for each } j=1,2,3, \ldots
$$

Let $\bar{\theta} \in[-\pi / 2, \pi / 2]$ be a limit point of the sequence $\left\{\theta_{+}\left(\alpha_{j}, \beta_{j}\right)\right\}$. Continuity of $g_{+}$is enough to conclude that

$$
g_{+}(\alpha, \beta, \bar{\theta})=0 .
$$

Since $(\alpha, \beta) \in R(I I)$, then $\bar{\theta}=\theta_{+}(\alpha, \beta)$, which is a contradiction.

Now let $\Gamma:[0,1] \rightarrow R(I I)$ be a path connecting $\left(\alpha_{1}, \beta_{1}\right)$ to $\left(\alpha_{2}, \beta_{2}\right)$. So $\Gamma(t)=(\alpha(t), \beta(t))$, where $\alpha(t)$ and $\beta(t)$ are continuous, $\Gamma(0)=\left(\alpha_{1}, \beta_{1}\right)$, and $\Gamma(1)=\left(\alpha_{2}, \beta_{2}\right)$. By continuity, $\theta_{+}(\Gamma[0,1])$ is a closed subinterval of $(-\pi / 2, \pi / 2)$. Let $\eta_{0}$ denote the left endpoint of this subinterval, i.e.,

$$
\eta_{0} \stackrel{\text { def }}{=} \min _{0 \leq t \leq 1} \theta_{+}(\Gamma(t)) \text {. }
$$

Also, set

$$
p \stackrel{\text { def }}{=} \min _{0 \leq t \leq 1} \sqrt{\beta(t)} \tan \eta_{0}
$$

and choose $\tilde{\theta} \in\left(-\pi / 2, \eta_{0}\right)$ such that

$$
\max _{0 \leq t \leq 1} \sqrt{\beta(t)} \tan \tilde{\theta}<p .
$$

Now let $q$ be chosen so that

$$
\max _{0 \leq t \leq 1} \sqrt{\beta(t)} \tan \tilde{\theta}<q<p .
$$

If $\mathscr{U} \subset C^{1}[0, \pi]$ is now defined by

$$
\mathscr{U} \stackrel{\text { def }}{=}\left\{u \in C^{1}[0, \pi] \mid \dot{u}(0)<q\right\},
$$


$\mathscr{U}$ is open $C^{1}[0, \pi]$. Now fix $R>0$ so that

$$
R>\max _{0 \leq t \leq 1}\|u(\cdot ; \Gamma(t), \tilde{\theta})\|_{1}
$$

then $\mathscr{N} \stackrel{\text { def }}{=} \mathscr{U} \cap B_{R}$ is a bounded open subset of $C^{1}[0, \pi]$ having the property that

$$
u_{+}(\cdot ; \Gamma(t), \widetilde{\theta}) \in \mathscr{N} \quad \text { for each } t \in[0,1]
$$

If $\theta(t)$ is used to denote the point such that

$$
\theta_{+}(\Gamma(t))<\theta(t)<\pi / 2 \quad \text { and } \sigma_{+}(\Gamma(t), \theta(t))=\sigma_{+}(\Gamma(t), \widetilde{\theta}), \quad 0 \leq t \leq 1,
$$

then

$$
u_{+}^{\prime}(0 ; \Gamma(t), \theta(t))=\sqrt{\beta(t)} \tan \theta(t)>\sqrt{\beta(t)} \tan \eta_{0} \geq p>q,
$$

whence

$$
u_{+}(\cdot ; \Gamma(t), \theta(t)) \notin \overline{\mathscr{N}} \text { for any } t \in[0,1]
$$

It follows that $u_{+}(\cdot ; \Gamma(t), \widetilde{\theta})$ is the unique solution of

$$
u=K\left[\alpha(t) u^{-}+\beta(t) u^{+}\right]+l_{t}, \quad u \in \overline{\mathscr{N}},
$$

for each $t \in[0,1]$, where

$$
l_{t}(x) \stackrel{\text { def }}{=}((\pi-x) / \pi)+\sigma_{+}(\Gamma(t), \tilde{\theta})(x / \pi), \quad 0 \leq x \leq \pi .
$$

Letting $t$ be a homotopy parameter, we can conclude that

$$
\operatorname{deg}_{L S}\left(\mathrm{id}-K\left[\alpha(t)(\cdot)^{-}+\beta(t)(\cdot)^{+}\right], \mathscr{N}, l_{t}\right)
$$

is independent of $t \in[0,1]$. Thus,

$$
\operatorname{deg}_{L S}\left(\mathrm{id}-K\left[\alpha_{1}(\cdot)^{-}+\beta_{1}(\cdot)^{+}\right], \mathscr{N}, l_{0}\right)=\operatorname{deg}_{L S}\left(\mathrm{id}-K\left[\alpha_{2}(\cdot)^{-}+\beta_{2}(\cdot)^{+}\right], \mathcal{N}, l_{1}\right)
$$

and hence

$$
\operatorname{ind}\left(u_{+}\left(\cdot ; \alpha_{1}, \beta_{1}, \tilde{\theta}\right)\right)=\operatorname{ind}\left(u_{+}\left(\cdot ; \alpha_{2}, \beta_{2}, \tilde{\theta}\right)\right)
$$

Since

$$
\tilde{\theta}<\eta_{0} \leq \min \left\{\theta_{+}\left(\alpha_{1}, \beta_{1}\right), \theta_{+}\left(\alpha_{2}, \beta_{2}\right) \dot{\}}\right.
$$

Lemma 10 implies that

$$
\operatorname{ind}\left(u_{+}\left(\cdot ; \alpha_{1}, \beta_{1}, \theta_{1}\right)\right)=\operatorname{ind}\left(u_{+}\left(\cdot ; \alpha_{1}, \beta_{1}, \tilde{\theta}\right)\right)
$$

whenever $\theta_{1}$ is such that $-\pi / 2<\theta_{1}<\theta_{+}\left(\alpha_{1}, \beta_{1}\right)$. Similarly,

$$
\operatorname{ind}\left(u_{+}\left(\cdot ; \alpha_{2}, \beta_{2}, \theta_{2}\right)\right)=\operatorname{ind}\left(u_{+}\left(\cdot ; \alpha_{2}, \beta_{2}, \tilde{\theta}\right)\right)
$$

whenever $\theta_{2}$ is such that $-\pi / 2<\theta_{2}<\theta_{+}\left(\alpha_{2}, \beta_{2}\right)$. So (64) now implies that

$$
\operatorname{ind}\left(u_{+}\left(\cdot ; \alpha_{1}, \beta_{1}, \theta_{1}\right)\right)=\operatorname{ind}\left(u_{+}\left(\cdot ; \alpha_{2}, \beta_{2}, \theta_{2}\right)\right)
$$

as desired. 
Theorem 12. If $(\alpha, \beta) \in R(I I)$ and $\theta<\theta_{+}(\alpha, \beta)$, then $\operatorname{ind}\left(u_{+}(\cdot ; \alpha, \beta, \theta)\right)=$ 1 .

Proof. From the previous two lemmas we need only find some point $(\alpha, \beta) \in$ $R(I I)$ and some $\theta<\theta_{+}(\alpha, \beta)$ such that

$$
\operatorname{ind}\left(u_{+}(\cdot ; \alpha, \beta, \theta)\right)=1 \text {. }
$$

To do this we will use Lemma 5, Proposition 6, and the index computation

$$
\operatorname{ind}\left(u_{+}(\cdot ; \alpha, \beta, \theta)\right)=1, \quad(\alpha, \beta) \in R(I), \theta \in(-\pi / 2, \pi / 2)
$$

obtained at the end of $\S 10$. The idea is to homotop this index across $\partial R(I) \cap$ $\partial R(I I)$ and into $R(I I)$.

As usual, we take $\left(\alpha_{0}, \beta_{0}\right)$ from $\partial R(I) \cap \partial R(I I)$ with $\alpha_{0}<\beta_{0}$. Fix $\sigma_{0}<-1$ and let $\theta_{0} \in(-\pi / 2, \pi / 2)$ be the unique point such that $\sigma_{+}\left(\alpha_{0}, \beta_{0}, \theta_{0}\right)=\sigma_{0}$. By Proposition 6 and Lemma 5 there exist a ball $B \subset C^{1}[0, \pi]$ centered at $u_{+}\left(\cdot ; \alpha_{0}, \beta_{0}, \theta_{0}\right)$ and a ball $B_{r_{0}}\left(\alpha_{0}, \beta_{0}\right) \subset Q^{+}$such that $u_{+}\left(\cdot ; \alpha, \beta, \theta_{0}\right) \in B$ is the unique solution of

$$
u=K\left[\alpha u^{-}+\beta u^{+}\right]+l(\alpha, \beta), \quad u \in \bar{B},
$$

where

$$
l(\alpha, \beta)(x) \stackrel{\text { def }}{=}((\pi-x) / \pi)+\sigma_{+}\left(\alpha, \beta, \theta_{0}\right)(x / \pi), \quad 0 \leq x \leq \pi,
$$

whenever $(\alpha, \beta) \in B_{r_{0}}\left(\alpha_{0}, \beta_{0}\right)$. Fix $\left(\alpha_{1}, \beta_{1}\right) \in B_{r_{0}}\left(\alpha_{0}, \beta_{0}\right) \cap R(I)$ and $\left(\alpha_{2}, \beta_{2}\right)$ $\in B_{r_{0}}\left(\alpha_{0}, \beta_{0}\right) \cap R(I I)$ and let $\Gamma:[0,1] \rightarrow B_{r_{0}}\left(\alpha_{0}, \beta_{0}\right)$ be a path connecting $\left(\alpha_{1}, \beta_{1}\right)$ to $\left(\alpha_{2}, \beta_{2}\right)$ and passing through the point $\left(\alpha_{0}, \beta_{0}\right)$. Then

$$
\operatorname{deg}_{L S}\left(\mathrm{id}-K\left[\alpha(t)(\cdot)^{-}+\beta(t)(\cdot)^{+}\right], B, l_{t}\right)=\mathrm{constant}
$$

for $0 \leq t \leq 1$, where $\Gamma(t)=(\alpha(t), \beta(t))$ and $l_{t}$ is defined by

$$
l_{t}(x) \stackrel{\text { def }}{=}((\pi-x) / \pi)+\sigma_{+}\left(\Gamma(t), \theta_{0}\right)(x / \pi), \quad 0 \leq x \leq \pi .
$$

Using (65) we have

$$
\begin{aligned}
1 & =\operatorname{ind}\left(u_{+}\left(\cdot ; \alpha_{1}, \beta_{1}, \theta_{0}\right)\right) \\
& =\operatorname{deg}_{L S}\left(\mathrm{id}-K\left[\alpha_{1}(\cdot)^{-}+\beta_{1}(\cdot)^{+}\right], B, l_{0}\right) \\
& =\operatorname{deg}_{L S}\left(\mathrm{id}-K\left[\alpha(t)(\cdot)^{-}+\beta(t)(\cdot)^{+}\right], B, l_{t}\right) \\
& =\operatorname{deg}_{L S}\left(\mathrm{id}-K\left[\alpha_{2}(\cdot)^{-}+\beta_{2}(\cdot)^{+}\right], B, l_{1}\right) \\
& =\operatorname{ind}\left(u_{+}\left(\cdot ; \alpha_{1}, \beta_{2}, \theta_{0}\right)\right) .
\end{aligned}
$$

Since $\theta_{0}<\theta_{+}\left(\alpha_{2}, \beta_{2}\right)$, Lemma 11 shows that

$$
\operatorname{ind}\left(u_{+}(\cdot ; \alpha, \beta, \theta)\right)=1
$$

whenever $(\alpha, \beta) \in R(I I)$ and $\theta<\theta_{+}(\alpha, \beta)$.

We are now in a position to compute the index of solutions corresponding to the right-hand branch of $\sigma_{+}(\alpha, \beta, \cdot)$ when $(\alpha, \beta) \in R(I I)$. 
Corollary 13. Fix $(\alpha, \beta) \in R(I I)$. Then

$$
\operatorname{ind}\left(u_{+}(\cdot ; \alpha, \beta, \theta)\right)=-1
$$

for all $\theta$ such that $\theta_{+}(\alpha, \beta)<\theta<\pi / 2$.

Proof. For such $(\alpha, \beta)$ we have seen that $\operatorname{ind}(0 ; \alpha, \beta)=0$. That is,

$$
\operatorname{deg}_{L S}\left(\text { id }-K\left[\alpha(\cdot)^{-}+\beta(\cdot)^{+}\right], B_{r}, 0\right)=0
$$

for each $r>0$. If $\theta$ is such that $\theta_{+}(\alpha, \beta)<\theta<\pi / 2$, we have only the two solutions

$$
u_{+}(\cdot ; \alpha, \beta, \theta) \text { and } u_{+}(\cdot ; \alpha, \beta, \widehat{\theta})
$$

of problem (3) with

$$
\sigma=\sigma_{+}(\alpha, \beta, \theta)=\sigma_{+}(\alpha, \beta, \widehat{\theta}),
$$

for some unique $\widehat{\theta}$ such that $-\pi / 2<\widehat{\theta}<\theta_{+}(\alpha, \beta)$. Choose $r>0$ sufficiently large that both of these solutions are to be found in $B_{r} \subset C^{1}[0, \pi]$. Then, using the additivity property of the Leray-Schauder degree, we see that

$$
\begin{aligned}
0= & \operatorname{deg}_{L S}\left(\mathrm{id}-K\left[\alpha(\cdot)^{-}+\beta(\cdot)^{+}\right], B_{r}, 0\right) \\
= & \operatorname{deg}_{L S}\left(\mathrm{id}-K\left[\alpha(\cdot)^{-}+\beta(\cdot)^{+}\right], B_{1}, 0\right) \\
& +\operatorname{deg}_{L S}\left(\mathrm{id}-K\left[\alpha(\cdot)^{-}+\beta(\cdot)^{+}\right], B_{2}, 0\right),
\end{aligned}
$$

where $B_{1}$ and $B_{2}$ are disjoint balls contained in $B_{r}$ and centered at $u_{+}(\cdot ; \alpha, \beta, \widehat{\theta})$ and $u_{+}(\cdot ; \alpha, \beta, \theta)$, respectively. By Theorem 12

$$
1=\operatorname{ind}\left(u_{+}(\cdot ; \alpha, \beta, \widehat{\theta})\right)=\operatorname{deg}_{L S}\left(\mathrm{id}-K\left[\alpha(\cdot)^{-}+\beta(\cdot)^{+}\right], B_{1}, 0\right)
$$

and hence

$$
-1=\operatorname{deg}_{L S}\left(\mathrm{id}-K\left[\alpha(\cdot)^{-}+\beta(\cdot)^{+}\right], B_{1}, 0\right)=\operatorname{ind}\left(u_{+}(\cdot, \alpha, \beta, \theta)\right) .
$$

In the proof of Theorem 12 we required that the path $\Gamma(t)$ pass through the point $\left(\alpha_{0}, \beta_{0}\right)$, so $\Gamma\left(t_{0}\right)=\left(\alpha_{0}, \beta_{0}\right)$ for some $t_{0} \in[0,1]$ and from (67) we have

$$
1=\operatorname{deg}_{L S}\left(\mathrm{id}=K\left[\alpha(t)(\cdot)^{-}+\beta(t)(\cdot)^{+}\right], B, l_{t_{0}}\right) .
$$

This implies that

$$
\operatorname{ind}\left(u_{+}\left(\cdot ; \alpha_{0}, \beta_{0}, \theta_{0}\right)\right)=1 .
$$

Corollary 14. Let $\left(\alpha_{0}, \beta_{0}\right) \in \partial R(I) \cap \partial R(I I)$ with $\alpha_{0}<\beta_{0}$. Then,

$$
\operatorname{ind}\left(u_{+}\left(\cdot, \alpha_{0}, \beta_{0}, \theta\right)\right)=1, \quad \theta \in(-\pi / 2, \pi / 2) .
$$

Theorem 12 and its corollaries are concerned with the solutions $u_{+}$obtained from the mapping $\sigma_{+}$but, with minor changes in notation, the arguments work for the solutions $u_{-}$associated with the map $\sigma_{-}$. Therefore we have the analogous results 
Theorem 15. If $(\alpha, \beta) \in R(I I)$ and $\theta<\theta_{-}(\alpha, \beta)$ then $\operatorname{ind}\left(u_{-}(\cdot ; \alpha, \beta, \theta)\right)=$ 1 .

Corollary 16. Fix $(\alpha, \beta) \in R(I I)$. Then

$$
\operatorname{ind}\left(u_{-}(\cdot ; \alpha, \beta, \theta)\right)=-1
$$

for all $\theta$. such that $\theta_{-}(\alpha, \beta)<\theta<\pi / 2$.

Corollary 17. Let $\left(\alpha_{0}, \beta_{0}\right) \in \partial R(I) \cap \partial R(I I)$ with $\alpha_{0}<\beta_{0}$. Then

$$
\operatorname{ind}\left(u_{-}\left(\cdot, \alpha_{0}, \beta_{0}\right)\right)=1, \quad \theta \in(-\pi / 2, \pi / 2) .
$$

Our approach in this section was to continue the index, ind $(0 ; \alpha, \beta)$, of the trivial solution from region $R(I)$, where this index was easily computed to be 1 , onto the "left branch" solutions associated with parameter values in region $R(I I)$. In this manner we were able to deduce the index of solutions associated with parameters located on the curve $\partial R(I) \cap \partial R(I I)$ (cf. Corollary 17). Of course, we could have started with $(\alpha, \beta)$ in $R(I I I)$, where $\operatorname{ind}(0 ; \alpha, \beta)=-1$, and continued this index to solultions associated with the "right-hand branch" of solutions in $R(I I)$. If this approach is taken then, again with only notational changes in the argument above for Corollary 14.

Corollary 18. Let $\left(\alpha_{0}, \beta_{0}\right) \in \partial R(I I) \cap \partial R(I I I)$ with $\alpha_{0}<\beta_{0}$. Then

$$
\operatorname{ind}\left(u_{ \pm}\left(\cdot, \alpha_{0}, \beta_{0}, \theta\right)\right)=-1, \quad \theta \in(-\pi / 2, \pi / 2) .
$$

if $(\alpha, \beta) \in R(I I)$ and $\sigma_{0}<0$, then from $\S 7.2$ we know that problem (4)

$$
\left\{\begin{array}{l}
u^{\prime \prime}+\alpha u^{-}+\beta u^{+}=0, \\
u(0)=0, \quad u(\pi)=\sigma_{0}
\end{array}\right.
$$

has exactly two solutions. In that section we call these solutions $w_{1}$ and $w_{2}$ and found that they were given by

$$
w_{1}(\cdot)=s_{1} u\left[\cdot ;\left(\begin{array}{l}
0 \\
1
\end{array}\right)\right] \text { and } w_{2}(\cdot)=s_{2} u\left[\cdot ;\left(\begin{array}{c}
0 \\
-1
\end{array}\right)\right]
$$

where the positive real numbers $s_{1}$ and $s_{2}$ were uniquely determined by the conditions

$$
s_{1} u\left[\pi ;\left(\begin{array}{l}
0 \\
1
\end{array}\right)\right]=\sigma_{0}=s_{2} u\left[\pi ;\left(\begin{array}{c}
0 \\
-1
\end{array}\right)\right] \text {. }
$$

Since

$$
\dot{w}_{1}(0)=s_{1} v\left[0 ;\left(\begin{array}{l}
0 \\
1
\end{array}\right)\right]=s_{1}>0
$$

and

$$
\dot{w}_{2}(0)=s_{2} v\left[0 ;\left(\begin{array}{c}
0 \\
-1
\end{array}\right)\right]=-s_{2}<0,
$$

we may conclude that each boundary condition $\sigma=\sigma_{0}<0$ uniquely determines the two solutions of problem (4) and that they are distinguished by their initial 
slopes, one being positive and the other negative. Now suppose that we use the notation

$$
w(\cdot ; \alpha, \beta, s) \stackrel{\text { def }}{=} \begin{cases}s u\left[\cdot ;\left(\begin{array}{l}
0 \\
1
\end{array}\right)\right], & s \geq 0, \\
-s u\left[\cdot ;\left(\begin{array}{c}
0 \\
-1
\end{array}\right)\right], & s<0 .\end{cases}
$$

With the above notation we always have

$$
\dot{w}\left(\cdot ; \alpha, \beta, s_{1}\right) \neq w\left(\cdot ; \alpha, \beta, s_{2}\right) \text { if } s_{1} \neq s_{2}
$$

and

$$
\dot{w}(0 ; \alpha, \beta, s)=s, \quad s \in \mathbf{R} .
$$

The family of solutions for problem (4), as $\sigma$ ranges over the nonpositive real axis, is then given by $\{w(\cdot ; \alpha, \beta, s)\}_{s \in \mathbf{R}}$.

Theorem 19. If $(\alpha, \beta) \in R(I I)$ or $\partial R(I) \cap R(I I)$ with $\alpha<\beta$ and $s<0$ then

$$
\operatorname{ind}(w(\cdot ; \alpha, \beta, s))=1
$$

and if $(\alpha, \beta) \in R(I I)$ or $\partial R(I I) \cap R(I I I)$ with $\alpha<\beta$ and $s>0$ then

$$
\operatorname{ind}(w(\cdot ; \alpha, \beta, s))=-1 \text {. }
$$

Proof. Suppose that $(\alpha, \beta) \in R(I I)$ is fixed. Then choose $(\bar{\alpha}, \bar{\beta})$ somewhere in $R(I)$ and define, for $t \in[0,1]$, the line segment

$$
\Gamma(t) \stackrel{\text { def }}{=}(1-t)(\bar{\alpha}, \bar{\beta})+t(\alpha, \beta)
$$

so $\Gamma$ connects $(\bar{\alpha}, \bar{\beta})$ to $(\alpha, \beta)$ and defines functions

$$
\alpha(t) \stackrel{\text { def }}{=}(1-t) \bar{\alpha}+t \alpha \quad \text { and } \quad \beta(t) \stackrel{\text { def }}{=}(1-t) \bar{\beta}+t \beta .
$$

Fix $s<0$ and choose $R$ so that

$$
R>\max _{t \in[0,1]}\|w(\cdot ; \Gamma(t), s)\|_{1} .
$$

Let $\varepsilon>0$ be sufficiently small that $s+\varepsilon<0$ and define

$$
l_{t}(x) \stackrel{\text { def }}{=}-s u\left[\pi ; \alpha(t), \beta(t),\left(\begin{array}{c}
0 \\
-1
\end{array}\right)\right]\left(\frac{x}{\pi}\right), \quad t \in[0,1] \text { and } 0 \leq x \leq \pi,
$$

where we have emphasized the dependence of the solution on the parameters $\alpha(t)$ and $\beta(t)$. Define the neighborhood $\mathcal{N}$ by

$$
\mathscr{N} \stackrel{\text { def }}{=} B_{R} \cap\left\{w \in C^{1}[0, \pi] \mid \dot{w}(0)<s+\varepsilon\right\} .
$$

Then $\mathscr{N}$ is a bounded open set in $C^{1}[0, \pi]$ such that, when $t \in[0,1]$, $w(\cdot ; \Gamma(t), s)$ is the unique solution of

$$
w=K\left[\alpha(t) w^{-}+\beta(t) w^{+}\right]+l_{t}
$$

in $\overline{\mathcal{N}}$; i.e., if $t$ is such that $\Gamma(t) \in R(I I)$ then $w(\cdot ; \Gamma(t), \widetilde{s}(t))$, for some unique $\tilde{s}(t)>0$, is the only other solution of (67) but it always has a positive initial 
slope and so cannot be in $\overline{\mathcal{N}}$. Again, by using $t$ as a homotopy parameter, we see that

$$
\begin{aligned}
\operatorname{ind}(w(\cdot, \bar{\alpha}, \bar{\beta}, s)) & =\operatorname{deg}_{L S}\left(\mathrm{id}-K\left[\alpha(t)(\cdot)^{-}+\beta(t)(\cdot)^{+}\right], \mathcal{N}, l_{t}\right), \quad t \in[0,1], \\
& =\operatorname{ind}(w(\cdot ; \alpha, \beta, s)) .
\end{aligned}
$$

As before, we may use the fact that

$$
\operatorname{ind}(0 ; \bar{\alpha}, \bar{\beta})=1
$$

to imply that

$$
\operatorname{ind}(w(\cdot, \bar{\alpha}, \bar{\beta}, s))=1 .
$$

Since the above argument holds if $(\alpha, \beta)$ belongs to $\partial R(I) \cap R(I I)$, the first assertion of the theorem is proved.

The argument above also works to prove the second assertion. We just choose $(\bar{\alpha}, \bar{\beta})$ from region $R(I I I)$ and use the index computation

$$
\operatorname{ind}(0 ; \widehat{\alpha}, \widehat{\beta})=-1,
$$

which holds whenever $(\widehat{\alpha}, \widehat{\beta})$ belongs to region $R(I I I)$.

We use these index computations to prove the following theorems concerning the perturbed problem

$$
\left\{\begin{array}{l}
u^{\prime \prime}+f(u)=h, \\
u(0)=\sigma_{1}, \quad u(\pi)=\sigma_{2} .
\end{array}\right.
$$

First, from Theorem 3, recall that the regions $\mathscr{O}_{2}$ and $\mathscr{O}_{0}$ were the two-solution and the zero-solution regions in the $\sigma_{1} \sigma_{2}$-plane for problem $(63)$ when $(\alpha, \beta) \in$ $R(I I)$.

Theorem 20. Fix $(\alpha, \beta) \in R(I I),\left(\sigma_{1}, \sigma_{2}\right) \in \mathscr{O}_{2}$, and $h \in C[0, \pi]$. Then there exists a positive real number $T_{0}$ such that the problem

$$
\left\{\begin{array}{l}
u^{\prime \prime}+f(u)=h, \\
u(0)=t \sigma_{1}, \quad u(\pi)=t \sigma_{2},
\end{array}\right.
$$

has at least two distinct solutions for all $t \geq T_{0}$. Moreover, if $\left(\sigma_{1}, \sigma_{2}\right) \in \mathscr{O}_{0}$, then there exists a positive real number $\widehat{T}_{0}$ such that the problem (68) has no solution if $t \geq \widehat{T}_{0}$.

Proof. Suppose that $\left(\sigma_{1}, \sigma_{2}\right) \in R(I I)$ with $\sigma_{1}>0$. Put $\sigma_{0}=\sigma_{2} / \sigma_{1}$ and let $\theta_{0}$ and $\hat{\theta}_{0}$ be the distinct points in the open interval $(-\pi / 2, \pi / 2)$ uniquely defined by

$$
\sigma_{+}\left(\alpha, \beta, \theta_{0}\right)=\sigma_{+}\left(\alpha, \beta, \widehat{\theta}_{0}\right)=\sigma_{0} .
$$

Thus, $u_{+}\left(\cdot ; \alpha, \beta, \theta_{0}\right)$ and $u_{+}\left(\cdot ; \alpha, \beta, \widehat{\theta}_{0}\right)$ are the only solutions of problem (3) with $\sigma=\sigma_{0}$. For $0 \leq x \leq \pi$ set

$$
l_{0}(x) \stackrel{\text { def }}{=}((\pi-x) / x)+\sigma_{0}(x / \pi) .
$$


Then there are two disjoint balls $B_{0}$ and $\widehat{B}_{0}$ contained in $C^{1}[0, \pi]$ and centered at $u_{+}\left(\cdot ; \alpha, \beta, \theta_{0}\right)$ and $u_{+}\left(\cdot ; \alpha, \beta, \widehat{\theta}_{0}\right)$, respectively, such that, from Theorem 12 and Corollary 13, we may conclude that

$$
\begin{aligned}
1 & =\operatorname{ind}\left(u\left(\cdot ; \alpha, \beta, \theta_{0}\right)\right) \\
& =\operatorname{deg}_{L S}\left(\operatorname{id}-K\left[\alpha(\cdot)^{-}+\beta(\cdot)^{+}\right], B_{0}, l_{0}\right)
\end{aligned}
$$

and

$$
\begin{aligned}
-1 & =\operatorname{ind}\left(u\left(\cdot ; \alpha, \beta, \widehat{\theta}_{0}\right)\right) \\
& =\operatorname{deg}_{L S}\left(\operatorname{id}-K\left[\alpha(\cdot)^{-}+\beta(\cdot)^{+}\right], \widehat{B}_{0}, l_{0}\right) .
\end{aligned}
$$

From Lemma 8, with $\tau \stackrel{\text { def }}{=} \sigma_{1} t$, we conclude that there exists a positive real number $\tau_{0}$ such that

$$
\operatorname{deg}_{L S}\left(\mathrm{id}-K\left[\alpha(\cdot)^{-}+\beta(\cdot)^{+}\right], B_{0}, l_{0}\right)=\operatorname{deg}_{L S}\left(\mathrm{id}-K\left[f_{\tau}(\cdot)-(1 / \tau) h\right], B_{0}, l_{0}\right)
$$

and

$$
\operatorname{deg}_{L S}\left(\mathrm{id}-K\left[\alpha(\cdot)^{-}+\beta(\cdot)^{+}\right], \widehat{B}_{0}, l_{0}\right)=\operatorname{deg}_{L S}\left(\mathrm{id}-K\left[f_{\tau}(\cdot)-(1 / \tau) h\right], \widehat{B}_{0}, l_{0}\right)
$$

whenever $\tau \geq \tau_{0}$. Therefore, there exist $u_{1} \in B_{0}$ and $u_{2} \in \widehat{B}_{0}$ such that

$$
\left\{\begin{array}{l}
u_{j}^{\prime \prime}+\alpha u_{j}^{-}+\beta u_{j}^{+}+\nu\left(t \sigma_{1} u_{j}\right) / t \sigma_{1}=\left(1 / t \sigma_{1}\right) h, \\
u_{j}(0)=1, \quad u_{j}(\pi)=\sigma_{0}=\sigma_{2} / \sigma_{1},
\end{array}\right.
$$

for $j=1,2$, whenever $t \geq \tau_{0} / \sigma_{1}$. Setting $\bar{u}_{j} \stackrel{\text { def }}{=} t \sigma_{1} u_{j}, j=1,2$, proves the first part of the theorem when $\sigma_{1}>0$.

In view of the index computations of Theorem 15 and Corollary 16 the case of $\sigma_{1}<0$ may be treated in the same fashion. Also, from Theorem 19 and the discussion preceding it, the case of $\sigma_{1}=0$ may be treated likewise.

The second assertion of this theorem is proved by contradiction. Fix $\left(\sigma_{1}, \sigma_{2}\right)$ $\in \mathscr{O}_{0}$ and assume that there exist a sequence of positive real numbers $\left\{t_{j}\right\}$ and a sequence $\left\{u_{j}\right\} \in C^{1}[0, \pi]$ with $t_{j} \rightarrow \infty$ and

$$
\left\{\begin{array}{l}
u_{j}^{\prime \prime}+f\left(u_{j}\right)=h, \\
u_{j}(0)=t_{j} \sigma_{1}, \quad u_{j}(\pi)=t_{j} \sigma_{2},
\end{array}\right.
$$

for each $j$. Since $\left\|u_{j}\right\|_{1} \geq \max \left\{t_{j} \sigma_{1}, t_{j} \sigma_{2}\right\}$, we see that $\left\|u_{j}\right\|_{1} \rightarrow \infty$ as $j \rightarrow \infty$. Furthermore, for each $j$,

$$
\frac{u_{j}}{\left\|u_{j}\right\|_{1}}=K\left[\frac{f\left(u_{j}\right)-h}{\left\|u_{j}\right\|_{1}}\right]+\frac{l_{j}}{\left\|u_{j}\right\|_{1}},
$$

where $l_{j}$ is defined by

$$
l_{j}(x) \stackrel{\text { def }}{=} t_{j} \sigma_{1}((\pi-x) / \pi)+t_{j}\left(\sigma_{2} x / \pi\right) .
$$

If $\left(\bar{\sigma}_{1}, \bar{\sigma}_{2}\right)$ is a limit point of the sequence

$$
\left\{\left(t_{j} \sigma_{1} /\left\|u_{j}\right\|_{1}, t_{j} \sigma_{2} /\left\|u_{j}\right\|_{1}\right)\right\}
$$


then either $\left(\bar{\sigma}_{1}, \bar{\sigma}_{2}\right) \in \mathscr{O}_{0}$ or $\left(\bar{\sigma}_{1}, \bar{\sigma}_{2}\right)=(0,0)$. Since $\left\{\left(f\left(u_{j}\right)-h\right) /\left\|u_{j}\right\|_{1}\right\}$ is bounded in $C[0, \pi]$, using the compactness of $K$ again we may assume that

$$
u_{j} /\left\|u_{j}\right\|_{1} \stackrel{C^{1}}{\rightarrow} v
$$

for some nontrivial $v \in C^{1}[0, \pi]$ satisfying

$$
v=K\left[\alpha v^{-}+\beta v^{+}\right]+\bar{l},
$$

where $\bar{l}$ is defined by

$$
\bar{l}(x) \stackrel{\text { def }}{=} \bar{\sigma}_{1}((\pi-x) / \pi)+\bar{\sigma}_{2}(x / \pi) .
$$

If $\left(\bar{\sigma}_{1}, \bar{\sigma}_{2}\right)=(0,0)$ then $v$ nontrivial contradicts $(69)$ having only the trivial solution, and if $\left(\bar{\sigma}_{1}, \bar{\sigma}_{2}\right) \in \mathscr{O}_{0}$ then (69) has no solution.

Our next and final result for the problem (68) deals with $(\alpha, \beta)$ in a resonance situation.

Theorem 21. Suppose that $(\alpha, \beta) \in \partial R(I) \cap \partial R(I I), \alpha<\beta$, or that $(\alpha, \beta) \in$ $\partial R(I I) \cap \partial R(I I I)$ with $\alpha<\beta$. Fix $\left(\sigma_{1}, \sigma_{2}\right)$ such that $\sigma_{2}<-\sigma_{1}$ and $h \in$ $C[0, \pi]$; then problem $(68)$ has a solution for all $t$ sufficiently large.

Proof. Recall that if $(\alpha, \beta)$ is on one of these curves, unique solutions for problem (63) exist whenever $\sigma_{2}<-\sigma_{1}$. Hence, we may use the index computations from Theorem 19 and Corollaries 14, 17, and 18 in the proof of Theorem 20 to prove this theorem.

\section{REFERENCES}

[AH] H. Amann and P. Hess, A multiplicity result for a class of elliptic boundary value problems, Proc. Roy Soc. Edinburgh Sect. A 84 (1979), 145-151.

[AP] A. Ambrosetti and G. Prodi, On the inversion of some differentiable mappings with singularities between Banach spaces, Ann. Mat. Pura Appl. 93 (1972), 231-246.

[BP] M. S. Berger and E. Podolak, On the solutions of a nonlinear Dirichlet problem, Indiana Univ. Math. J. 24 (1975), 837-846.

[Da1] E. N. Dancer, On the Dirichlet problem for weakly coupled nonlinear elliptic partial differential equations, Proc. Roy. Soc. Edinburgh Sect. A 76 (1977), 283-300.

[Da2] _ On the ranges of certain weakly nonlinear elliptic partial differential equations, $\mathrm{J}$. Math. Pures Appl. 57 (1978), 351-366.

[F1] S. Fučik, Boundary value problems with jumping nonlinearities, Časopis Pěst. Mat. 101 (1976), 69-87.

[F2] _ Solvability of nonlinear equations and boundary value problems, Boll. Un. Mat. Ital. 11 (1975), 250-267.

[F3] _ Remarks on a result of A. Ambrosetti and G. Prodi, Reidel, Dordrecht, 1980.

[Hs] G. A. Harris, Semilinear elliptic problems with nonhomogeneous boundary conditions, Ph. D. Dissertation, University of Utah, Salt Lake City, August 1987.

[Ha] P. Hartman, Ordinary differential equations, Birkhäuser, Boston, Mass., 1982.

[He] P. Hess, On a nonlinear elliptic boundary value problem of Ambrosetti-Prodi type, Boll. Un. Mat. Ital. 17 (1980), 187-192.

[LMc1] A. Lazer and P. McKenna, On the number of solutions of a nonlinear Dirichlet problem, J. Math. Anal. Appl. 87 (1981), 282-294. 
[LMc2] __ New multiplicity results for a class of semilinear elliptic and parabolic boundary value problems, J. Math. Anal. Appl. 107 (1985), 371-395.

[LMc3] __ On a conjecture related to the number of solutions of a nonlinear Dirichlet problem, Proc. Roy. Soc. Edinburgh Sect. A 85 (1983), 275-283.

[N] L. Nirenberg, Topics in nonlinear functional analysis, N.Y.U. Lecture Notes, 1973-1974.

[PS] H. O. Peitgen and K. Schmitt, Global analysis of two-parameter elliptic eigenvalue problems, Trans. Amer. Math. Soc. 283 (1984), 57-95.

[Sc1] K. Schmitt, Boundary value problems with jumping nonlinearities, Rocky Mountain J. Math. 16 (1986), 481-496.

[Sc2] _ Boundary value problems for quasilinear second order elliptic equations, J. Nonlinear Anal. 2 (1978), 263-309.

[Sc3] _ A Study of eigenvalue and bifurcation problems for nonlinear elliptic partial differential equations via topological continuation methods, Notes de Séminaire de Mathématique, CABAY, Louvain-la-Neuve, Belgium, 1982.

[So] S. Solimini, Existence of a third solution to a class of boundary value problems with jumping nonlinearities, Nonlinear Anal. TMA 7 (1983), 917-927.

Department of Mathematics (ACA), Auburn University, Auburn, Alabama 36849 